\title{
Ocneanu Cell Calculus and Integrable Lattice Models
}

\author{
Ph. Roche \\ Centre de Physique Theorique, Ecole Polytechnique, F-91128 Palaiseau Cedex, France
}

\begin{abstract}
We show that cell calculus (first introduced by Ocneanu in the context of relative position of factors) is a technique which permits us to connect different integrable models. It generalizes the Vertex-IRF correspondence.
\end{abstract}

Since the work of Baxter [Ba1], the number of integrable lattice models (ILM) has increased rapidly. Besides, conformal field theory (CFT) [BPZ, Gi1], full of success, has reached a status where the problem of classifying all CFT is worth being investigated [MS]. In a similar way, it is time to put order in the huge amount of solutions of the Yang-Baxter equation (YBE).

Quantum groups [Ji1, Dr] realize part of this: any highest weight representation of the deformation of a simple Lie algebra yields a solution of YBE. Unfortunately all solutions obtained this way are trigonometric functions of the spectral parameter. Except for the 8 Vertex model, which is associated to the Sklyanin algebra [Sk] [an elliptic deformation of $S U(2)$ ], one does not know what the algebraic objects associated to elliptic solutions of YBE are. We should add for completeness that there exist solutions of $\mathrm{YBE}$ which are related to algebraic curve of genus greater than $2[\mathrm{Au}-\mathrm{Y}, \mathrm{Ba} 2]$.

In this short introduction we will restrict ourselves to integrable critical models associated to CFT having central charge $c \leqq 1$ because one knows lots of things in this case [Pa.1, Gi.2]. Representation of spin $\frac{1}{2}$ of $\mathscr{U S U}(2)_{q}$ leads to the 6 Vertex model. Using an IRF-Vertex transformation one obtains an SOS model which can be consistently modified to a RSOS model when $q$ is a root of unity [ABF, Pa.2]. These last models are the ABF models based on the Dynkin diagram $A_{n}$. This method does not shed any light on the construction of Pasquier models based on other Dynkin diagrams. On the other hand it would be an important step if one could understand more abstractly how ADE models are built.

In [FG] it has been shown that $D_{n}$ lattice models can be constructed from the $A_{n}$ lattice models via a discrete symmetry $Z_{2}$. Using a different point of view, we show that on the lattice these models are linked via an object called a cell system 
[Oc, Pa.3]. This connection works as well away from the critical point. We also show that $E_{6}$ models are related to $A_{11}$ models via a cell system.

In the $c=1$ case, Pasquier has built models associated to each extended Dynkin diagram. All these models can be easily constructed from an IRF-Vertex correspondence using cells which are Clebsch-Gordan coefficients of the subgroups of $S U(2)$. All these features can be generalized using the work of Ocneanu $[\mathrm{Oc}]$ on inclusion of hyperfinite factors [Su]. Cell calculus is shown to be a tool for classifying solutions of YBE.

The paper is organized as follows: In the first part we describe cell calculus using Ocneanu results. We show why it is relevant for physics and we give a few examples. In the second part we describe an important class of cell systems, namely those which are associated to group theory. As an example we show how to obtain Pasquier's models built on extended Dynkin diagram and we construct new models.

After this work was completed, we received a paper of DiFrancesco and Zuber [DZ] which overlaps substantially with our present work. They have used Cell Calculus to construct new models from $S U(N)$ lattice integrable models. They have found a very interesting connection between the set of intertwining matrices (called $C$ in our work) and the fusion algebra of WZW models, and they have noticed a generalisation of MacKay correspondence between subgroups of $S U(3)$ and incidence matrices of restricted height models.

\section{String Algebras and Cell Calculus}

This part is devoted to an introduction for physicists to the deep and difficult theory of Ocneanu [Oc]. Most of the concepts are taken from Ocneanu's papers and Pasquier's thesis [Pa.3]. However the introduction of local operators and the proof of identity of partition functions on a cylinder of various statistical models definitely prove why these constructions are important in 2-dim physics.

Path and String Algebras. The set of known ILM can be roughly divided in two families: vertex models and IRF (interaction round faces) models. We recall their definitions and show that they can be unified in the same formalism by introducing graphs.

Vertex models are statistical models, defined on a square lattice. Microscopic variables are located on the edges of the lattice. They interact via a vertex. If $\alpha, \beta, \gamma, \delta$ are the states of edges surrounding a fixed vertex, the Boltzmann weight associated to this configuration is $W(\alpha, \beta \mid \gamma, \delta)$ (Fig. 1). Boltzmann weights depend on an additional parameter (called spectral parameter) which controls anisotropy of the system. In this representation YBE can be written as:

$$
\begin{aligned}
& \sum_{\alpha_{2}, \beta_{2}, \gamma_{2}} W\left(\alpha_{1}, \beta_{2} \mid \beta_{3}, \alpha_{2}\right) W^{\prime}\left(\alpha_{2}, \gamma_{2} \mid \gamma_{3}, \alpha_{3}\right) W^{\prime \prime}\left(\gamma_{1}, \beta_{1} \mid \beta_{2}, \gamma_{2}\right) \\
& \quad=\sum_{\alpha_{2}, \beta_{2}, \gamma_{2}} W^{\prime \prime}\left(\gamma_{2}, \beta_{2} \mid \beta_{3}, \gamma_{3}\right) W^{\prime}\left(\alpha_{1}, \gamma_{1} \mid \gamma_{2}, \alpha_{2}\right) W\left(\alpha_{2}, \beta_{1} \mid \beta_{2}, \alpha_{3}\right) .
\end{aligned}
$$

Fig. 1

$$
W(\alpha, \beta \mid \gamma, \delta)=\alpha-\left.\right|^{\gamma} \delta
$$




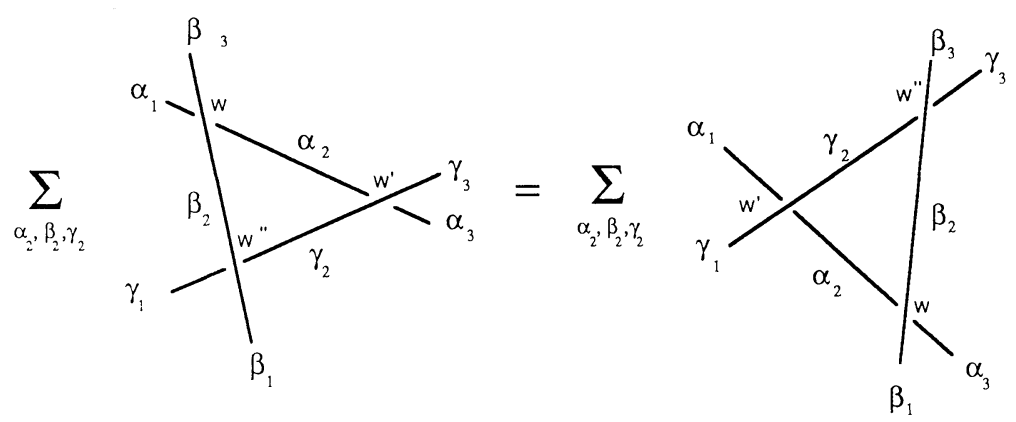

Fig. 2

Fig. 3 a
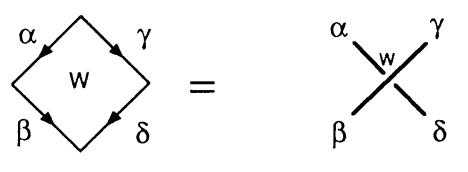

Fig. 3 b

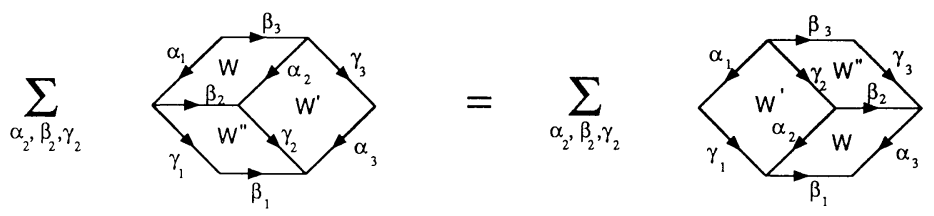

$$
w(a, b, c, d)=b<d
$$

Fig. 4

This formula is conveniently written in a picture (Fig. 2) which is reminiscent of the third Reidermeister move in knot theory. We prefer for further purposes that edges interact via faces (Fig. 3). These two representations are equivalent after a change of the lattice and its dual. Arrows along edges have no relation with states of the edge but merely indicate an orientation. Because in general there is no rotational symmetry, i.e. $W(\alpha, \beta \mid \gamma, \delta) \neq W(\gamma, \alpha \mid \delta, \beta)$, these arrows prevent us making mistakes in graphical proofs. The convention taken here is arrows pointing down.

The simplest vertex ILM is the 6 vertex model, generalized in the 8 vertex. Other integrable vertex models can be constructed via fusion methods [KRS, DJKMO]. These models are associated to quantum $S U(2)$; other models associated to other quantum groups have been built.

In the IRF case, microscopic variables (heights) are located on the nodes of the square lattice and interact via face. If $a, b, c, d$ are the value of heights surrounding a face, the Boltzmann weight attached to it is $W(a, b, c, d)$ (Fig. 4). We have put arrows on the edges of the square to indicate an orientation of the square (due to a lack of rotational symmetry). In the case of ABF models or Pasquier's models, heights take their value in a Dynkin diagram such that $(a, b),(b, c),(c, d),(d, a)$ are neighbours on the diagram. 
Fig. 5
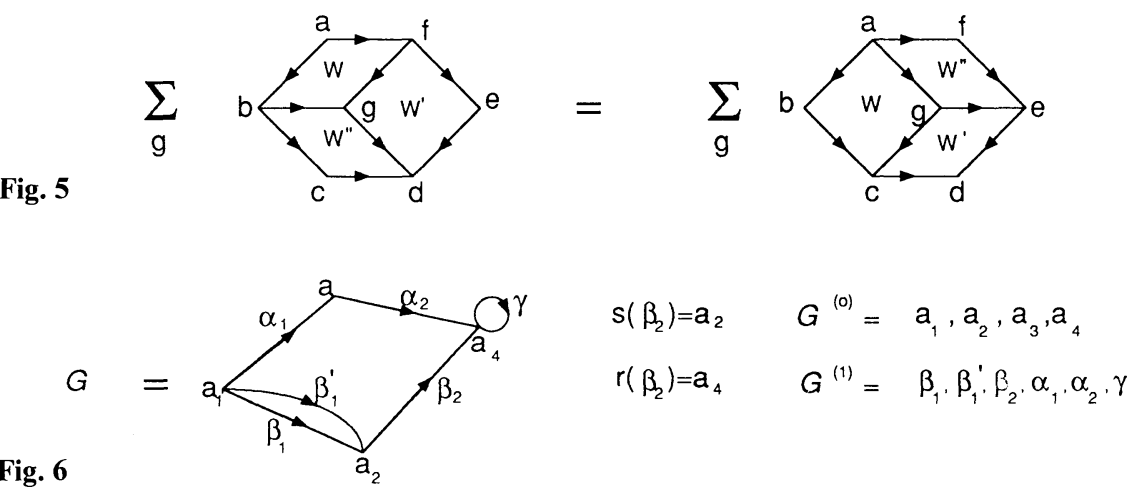

Fig. 7

$$
W\left(\beta_{1}, \beta_{2} \mid \alpha_{1}, \alpha_{2}\right)=\left\langle\sum_{\alpha_{2}}^{\alpha_{1}}=\sum_{\alpha_{2}}^{\alpha_{1}}=\sum_{\alpha_{2}}^{a_{1}}\right.
$$

YBE reads in this representation:

$$
\begin{aligned}
\sum_{g} W(a, b, g, f) W^{\prime}(f, g, d, e) W^{\prime \prime}(b, c, d, g) \\
\quad=\sum_{g} W^{\prime \prime}(a, g, e, f) W^{\prime}(a, b, c, g) W(g, c, d, e)
\end{aligned}
$$

(Fig. 5). It is now easy to put vertex models and IRF models in the same formalism. Let $\mathscr{G}$ be a graph (oriented or not). Let $\mathscr{G}^{(0)}$ be the set of nodes of $\mathscr{G}$ and $\mathscr{G}^{(1)}$ the set of arrows in $\mathscr{G}$. We will use roman letters for elements of $\mathscr{G}^{(0)}$ and greek letters for elements of $\mathscr{G}^{(1)}$. If $\alpha$ is an arrow, we call $s(\alpha)$ its source and $r(\alpha)$ its range, i.e. $\alpha$ goes from $s(\alpha)$ to $r(\alpha)$ (Fig. 6).

In the paper we will define integrable models associated to certain graphs. These models will be defined by putting elements of $\mathscr{G}^{(1)}$ on oriented edges of the square lattice. They will interact around faces. Let $W(\alpha, \beta \mid \gamma, \delta)$ be the Boltzmann weight associated to the configuration described in Fig. 7. Configurations of arrows should satisfy the following rule:

$$
s(\alpha)=s(\gamma), \quad r(\alpha)=s(\beta), \quad r(\gamma)=s(\delta), \quad r(\beta)=r(\delta) .
$$

Although redundant it will be sometimes useful to use the following picture for Boltzmann weights (Fig. 7).

When $\mathscr{G}^{(0)}$ is restricted to a point we obtain ordinary vertex models.

When $\mathscr{G}$ is non-oriented and such that there is at most one arrow from one point to another, we recover ordinary IRF models.

We now introduce a few definitions.

A path of length $n \geqq 1$ is a $n$-uplet $\varrho=\left(\alpha_{1}, \ldots, \alpha_{n}\right)$ of elements of $\mathscr{G}^{(1)}$ such that: $r\left(\alpha_{i}\right)=s\left(\alpha_{i+1}\right)$ for $1 \leqq i \leqq n-1$. We define $l(\varrho)=n$ the length of $\varrho$; the path $\varrho$ has source $s(\varrho)=s\left(\alpha_{1}\right)$ and range $r(\varrho)=r\left(\alpha_{n}\right)$. 
Fig. 8

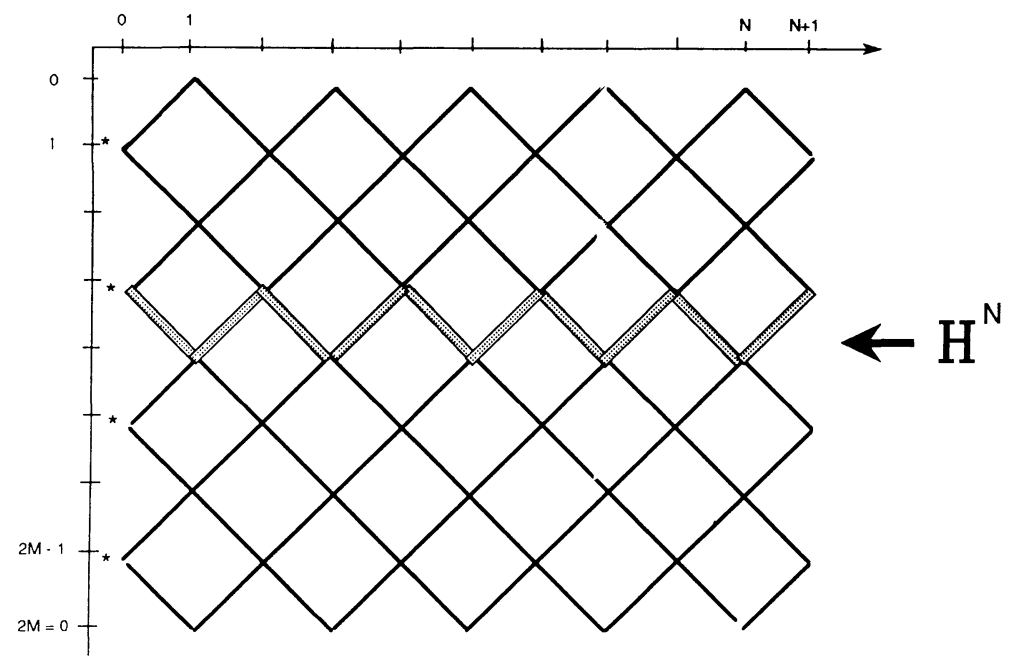

Let Path ${ }_{x, y}^{n}$ be the set of paths of length $n$ going from $x$ to $y$. It will be useful to introduce the following notations:

$$
\operatorname{Path}_{x}^{n}=\bigcup_{y} \operatorname{Path}_{x, y}^{n}
$$

and

$$
\operatorname{Path}_{x, y}=\bigcup_{n} \operatorname{Path}_{x, y}^{n} .
$$

There is an obvious composition law on paths: if $\varrho=\left(\alpha_{1}, \ldots, \alpha_{n}\right)$ and $\sigma=\left(\beta_{1}, \ldots, \beta_{m}\right)$, and if $r(\varrho)=s(\sigma)$, the composition of $\varrho$ and $\sigma$ is the $n+m$-uplet $\varrho \circ \sigma=\left(\alpha_{1}, \ldots, \alpha_{n}\right.$, $\left.\beta_{1}, \ldots, \beta_{m}\right)$. We will require that there exists a distinguished node $*$ such that for every $x$ in $\mathscr{G}^{(0)} \operatorname{Path}_{*, x} \neq \emptyset$.

We now define abstractly our statistical model and we will focus on algebras associated to it. Consider a square lattice with $2 M$ lines and $N+1$ columns $(N$ odd).

We choose for orientation left to right. We will use periodic conditions in the south-north direction and we will fix the value of the sites of the first column to be * (Fig. 8). The Hilbert space of our model is

$$
\mathscr{H}^{N}=\underset{\varrho \in \operatorname{Path}_{*}^{N}}{\bigoplus} \mathbb{C} \varrho .
$$

For convenience we introduce the space

$$
\mathscr{H}_{x}^{N}=\underset{\varrho \in \operatorname{Path}_{*}^{N}, x}{\oplus} \mathbb{C} \varrho .
$$

Let $\mathscr{A}_{x}^{N}, \mathscr{A}^{N}$ be the finite dimensional algebras defined by $\mathscr{A}_{x}^{N}=\operatorname{End}\left(\mathscr{H}_{x}^{N}, \mathscr{H}_{x}^{N}\right)$ and $\mathscr{A}^{N}=\bigoplus_{x} \mathscr{A}_{x}^{N}$. [We will also use the notation $\mathscr{A}^{N}(\mathscr{G})$.]

A string is a couple $\left(\varrho_{+}, \varrho_{-}\right) \in$ Path $_{*, x}$. The vector space $\mathscr{A}_{x}^{N}$ admits a basis labelled by strings $\left(\varrho_{+}, \varrho_{-}\right)$such that its action on the basis of $\mathscr{H}_{x}^{N}$ reads: $\left(\varrho_{+}, \varrho_{-}\right)$. $\gamma=\delta_{\varrho_{-}, \gamma} \varrho_{+}$. As a result we get the following algebra law for strings:

$$
\left(\varrho_{+}, \varrho_{-}\right)\left(\gamma_{+}, \gamma_{-}\right)=\delta_{\varrho_{-}, \gamma_{+}}\left(\varrho_{+}, \gamma_{-}\right) \text {. }
$$


Fig. 9
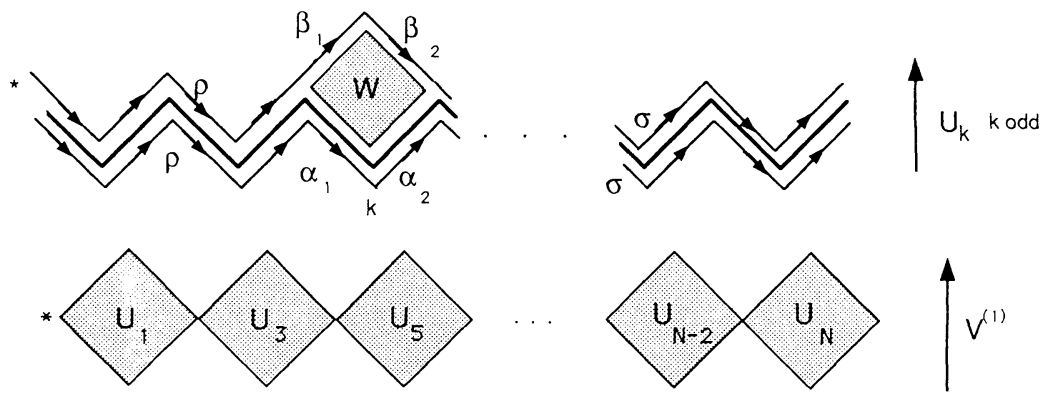

Fig. 10
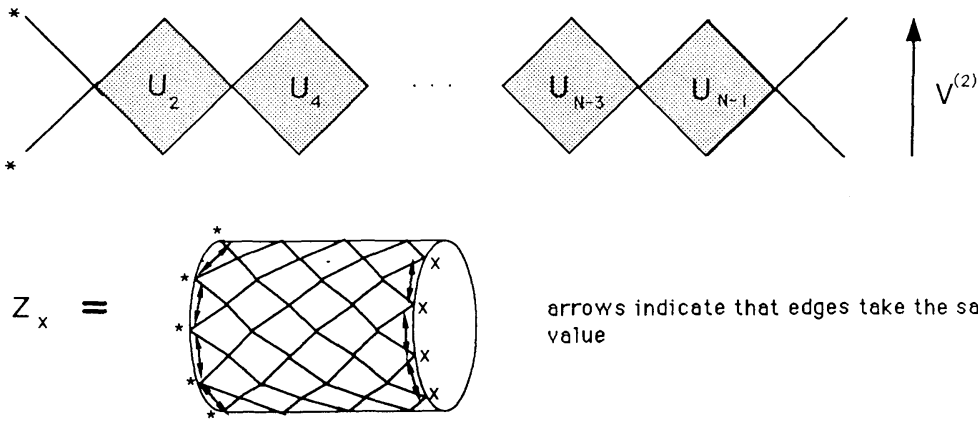

orrows indicate that edges take the same value

Fig. 11

These algebras are naturally endowed with an antilinear involutive operator $\dagger$ defined on string by: $\left(\varrho_{+}, \varrho_{-}\right)^{\dagger}=\left(\varrho_{-}, \varrho_{+}\right)$.

$\mathscr{A}_{x}^{N}, \mathscr{A}^{N}$ are called string algebras. They possess nice properties as we will see.

Using Boltzmann weights we can construct transfer matrices built from face operators. Face operators are operators which add one Boltzmann weight to a line at site $k$. For $k \leqq N-1$ the face operator $U_{k}$ acting on site $k$ is defined by:

$$
U_{k}=\sum_{\varrho, \sigma, \alpha_{1}, \alpha_{2}, \beta_{1}, \beta_{2}}\left(\varrho \circ \beta_{1} \circ \beta_{2} \circ \sigma, \varrho \circ \alpha_{1} \circ \alpha_{2} \circ \sigma\right) W\left(\beta_{1}, \beta_{2} \mid \alpha_{1}, \alpha_{2}\right) \text {, }
$$

where $\varrho, \sigma, \alpha_{1}, \alpha_{2}, \beta_{1}, \beta_{2}$ have to satisfy: $\varrho \in \operatorname{Path}_{*}^{k-1}, \alpha_{1}, \alpha_{2}, \beta_{1}, \beta_{2} \in \mathscr{G}^{(1)}$, $\sigma \in \mathrm{Path}^{N-k-1}, \quad s\left(\alpha_{1}\right)=s\left(\beta_{1}\right)=r(\varrho), \quad r\left(\alpha_{1}\right)=s\left(\alpha_{2}\right), \quad r\left(\beta_{1}\right)=s\left(\beta_{2}\right), \quad r\left(\alpha_{2}\right)=r\left(\beta_{2}\right)=s(\sigma)$ (Fig. 9).

Transfer matrices $V^{(1)}$ and $V^{(2)}($ Fig. 10) are defined by:

$$
V^{(1)}=\prod_{k \text { odd }} U_{k} \quad \text { and } \quad V^{(2)}=\prod_{k \text { even }} U_{k} .
$$

Let $Z_{x}$ be the partition function of this model on a cylinder with sites on the last column taking $x$ value (Fig. 11). $V^{(1)}$ and $V^{(2)}$ are elements of $\mathscr{A}^{N}$. We have:

$$
\operatorname{tr}\left(\left(V^{(1)} V^{(2)}\right)^{M}\right)=\sum_{x} Z_{x}
$$

We have in mind to handle the thermodynamic limit. String algebras have been introduced for this reason.

Proposition 1 (Ocneanu). Let us define a linear map $i_{p}^{q}: \mathscr{A}^{p} \mapsto \mathscr{A}^{q}$ by

$$
i_{p}^{q}\left(\varrho_{+}, \varrho_{-}\right)=\sum_{\sigma, l(\sigma)=q-p, s(\sigma)=r(\varrho)}\left(\varrho_{+} \circ \sigma, \varrho_{-} \circ \sigma\right) .
$$


This is a †-algebra morphism. Furthermore $i_{q}^{r} \circ i_{p}^{q}=i_{p}^{r}$, when $p \leqq q \leqq r$.

This allows us to define $\mathscr{A}=\lim _{\rightarrow} \mathscr{A}^{N}$.

All this needs just a trivial check.

Ocneanu has defined traces on string algebras which are useful for taking the thermodynamic limit. Let $\left(G_{x, y}\right)_{x, y \in \mathscr{G}(0)}$ be the matrix defined by $\left(G_{x, y}\right)=\operatorname{card}\left(\operatorname{Path}_{x, y}^{1}\right)$. This matrix is the incidence matrix of the graph $\mathscr{G}$. In the following we restrict ourselves to a graph having irreducible incidence matrix $[\mathrm{Ga}]$. When the graph is not oriented this means that the graph is connected. From the Perron-Frobenius theorem $G$ admits a positive non-degenerate eigenvalue $\beta$ which exceeds in module all the others. Moreover the eigenvector associated to $\beta$ can be chosen such that all its components are strictly positive and this is the only eigenvector satisfying this last requirement. Let $\left(S_{x}\right)_{x \in \mathscr{G}(0)}$ be this eigenvector. We impose $S_{*}=1$.

Proposition 2 (Ocneanu). Let $\operatorname{Tr}$ be the linear form on $\mathscr{A}^{N}$ defined by:

$$
\operatorname{Tr}\left(\varrho_{+}, \varrho_{-}\right)=\delta_{\varrho_{+}, \varrho_{-}} \beta^{-N} S_{r\left(\varrho_{+}\right)} .
$$

It satisfies: $\operatorname{Tr} \circ i_{m}^{N}=\operatorname{Tr}$ for $m \leqq N$,

$\operatorname{Tr}(\varrho \sigma)=\operatorname{Tr}(\sigma \varrho)$ for $\varrho, \sigma$ elements of $\mathscr{A}^{N}$,

$\operatorname{Tr}(1)=1$,

$\operatorname{Tr}\left(\varrho \varrho^{\dagger}\right) \geqq 0$ and $\operatorname{Tr}\left(\varrho \varrho^{\dagger}\right)=0 \Leftrightarrow \varrho=0$.

Proof. Simple checks.

The essential point for future applications is that we can to some extent replace the ordinary trace of matrices by Ocneanu's one in formula (1). This modification will not change the bulk energy of our system as well as the central charge if the system is at a critical point. More precisely, let $Z^{\prime}=\operatorname{Tr}\left(\left(V^{(1)} V^{(2)}\right)^{M}\right)$, it follows that:

$$
Z^{\prime}=\beta^{-N} \sum_{x} Z_{x} S_{x} .
$$

We will see how one can use this relation in the next part devoted to cell systems.

Cell Systems. Cell systems originally arise in the context of the study of the relative position of an hyperfinite factor in another one [Jo.1, Oc]. Here we will use cell systems to compare two different statistical models associated to different graphs. The mathematical objects we use are those constructed by Ocneanu. The idea underlying the following part is that if a model possesses discrete symmetries, it is possible to construct a new model which possesses the same specific features (integrability, same bulk free energy, same central charge if one model is scale invariant), but which is different at the level of operator content. This method seems to be the lattice analogue of construction of orbifold models in CFT [FG].

Let $\mathscr{G}_{1}$ and $\mathscr{G}_{2}$ be two graphs with distinguished nodes $*_{1}$ and $*_{2}$. Let $G_{1}$ and $G_{2}$ be their incidence matrices. We say that there exists a symmetry from $\mathscr{G}_{1}$ to $\mathscr{G}_{2}$ if there exists a card $\left(\mathscr{G}_{1}^{(0)}\right) \times \operatorname{card}\left(\mathscr{G}_{2}^{(0)}\right)$ matrix $C$ with nonnegative integer coefficients, such that:

$$
G_{1} C=C G_{2}, \quad C_{*_{1}, i}=1 \Leftrightarrow i=*_{2}, \quad \forall i, \exists j, C_{i, j} \neq 0 .
$$




$$
G_{1}=\stackrel{a_{1}}{b_{1}} \quad c_{1} \quad b_{1}^{b_{1}} \quad a_{1}^{a_{1}}
$$

$\left.1^{\circ}\right)$

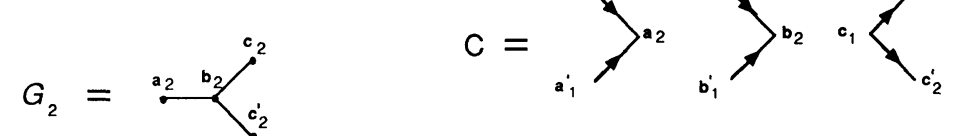

$\left.2^{\circ}\right)$

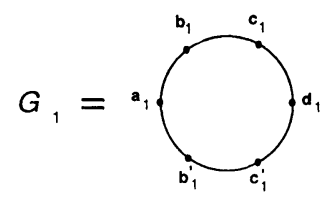

$$
G_{2}=\sum_{a_{2}^{\prime}}^{a_{2}} \sum_{d_{2}}^{b_{2} d_{2}}
$$

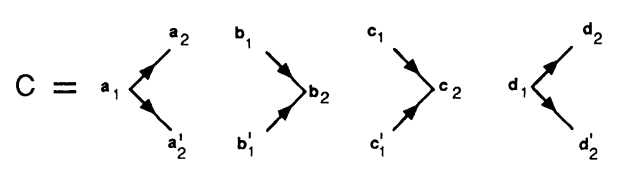

$\left.3^{\circ}\right)$

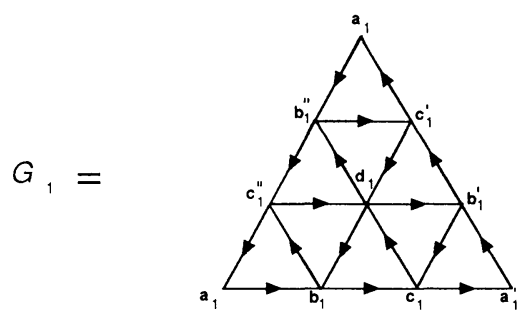

Fig. 12
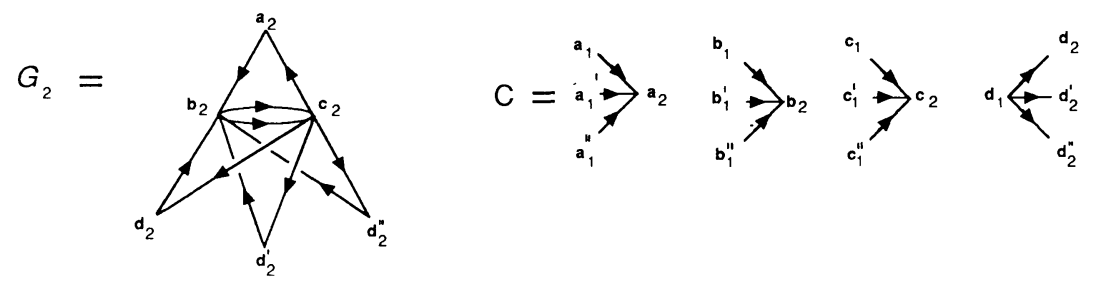

It is clear that the two matrices $G_{1}$ and $G_{2}$ share the same largest eigenvalues. Indeed, let $S$ be the eigenvector associated to the largest eigenvalue $\beta$ of $G_{2}$, i.e. $G_{2} S=\beta S$. Applying $C$ to this equation we get: $G_{1} C S=\beta C S$. Because $C S$ has just positive coefficients, it is the Perron-Frobenius vector of $G_{1}$ associated to the greatest eigenvalue $\beta$.

The matrix $C$ defines a graph $\mathscr{C}$. We define $\mathscr{C}^{(0)}=\mathscr{G}_{1}^{(0)} \cup \mathscr{G}_{2}^{(0)}$ and $\operatorname{Path}_{x, y}^{1}(\mathscr{C})=\emptyset$ if $x, y$ are both in $\mathscr{G}_{1}^{(0)}$ or in $\mathscr{G}_{1}^{(0)}$, and $\operatorname{card} \operatorname{Path}_{x, y}^{(1)}(\mathscr{C})=C_{x, y}$. See Fig. 12 for three illuminating examples.

A cell system is a family of complex numbers labelled by 4 arrows $\alpha, \alpha^{\prime}, \beta_{1}, \beta_{2}$ and pictured as follows:

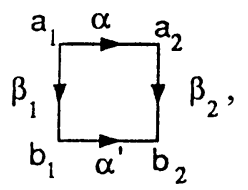


where $\alpha, \quad \alpha^{\prime} \in \mathscr{C}^{(1)} ; \quad \beta_{1} \in \mathscr{G}_{1}^{(1)} ; \beta_{2} \in \mathscr{G}_{2}^{(1)}$ and $s(\alpha)=s\left(\beta_{1}\right)=a_{1}, \quad r(\alpha)=s\left(\beta_{2}\right)=a_{2}$, $r\left(\beta_{2}\right)=r\left(\alpha^{\prime}\right)=b_{2}, r\left(\beta_{1}\right)=s\left(\alpha^{\prime}\right)=b_{1}$. Moreover this family should satisfy two nontrivial families of algebraic equations $(U$ and $T)$ we will introduce later. We remark that if $a_{1}$ and $b_{2}$ are fixed, the number of paths $\beta_{1} \circ \alpha^{\prime}$ going from $a_{1}$ to $b_{2}$ is equal to the number of paths $\alpha \circ \beta_{2}$ going from $a_{1}$ to $b_{2}$ because of the identity $G_{1} C=C G_{2}$. The convention we use is that any cell can be rotated in the plane and conserves its value:

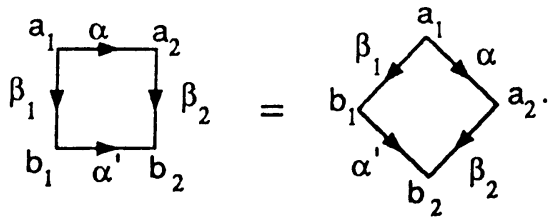

After a reflection the value of the cell is changed into its conjugate:

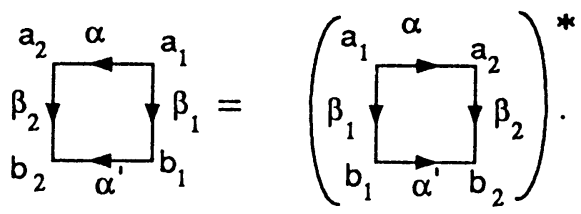

The following propositions are the heart of the matter.

Proposition 3 (Ocneanu). Algebra Morphism and Condition $(\boldsymbol{U})$. If the cell system satisfies the following condition (Unitarity),

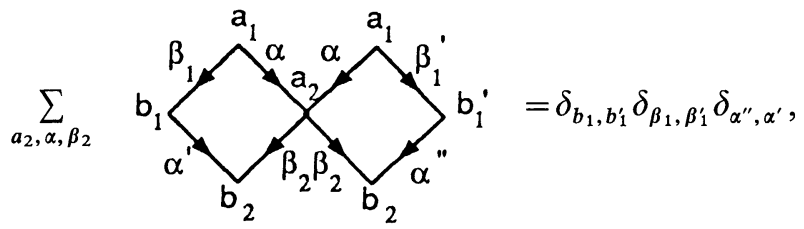

then the linear map $\varphi^{(N)}: \mathscr{A}^{N}\left(\mathscr{G}_{1}\right) \mapsto \mathscr{A}^{N}\left(\mathscr{G}_{2}\right)$ defined by:

$\varphi^{(N)}\left(\varrho_{+}, \varrho_{-}\right)=$

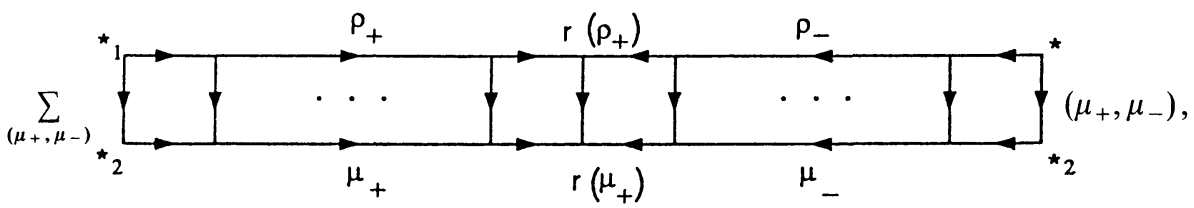

is a †-algebra morphism satisfying $\varphi^{(q)} \circ i_{p}^{q}=i_{p}^{q} \circ \varphi^{(p)}$. In the definition of $\varphi^{(N)}\left(\varrho_{+}, \varrho_{-}\right)$ there is an implicit sum over vertical arrows. We will use this convention all throughout the paper.

Proof. It is easy using graphical methods. It is given in the appendix and is worth looking at.

Remark 1. The last equation permits us to define

$$
\varphi=\lim _{\rightarrow} \varphi^{(N)}: \mathscr{A}\left(\mathscr{G}_{1}\right) \mapsto \mathscr{A}\left(\mathscr{G}_{2}\right)
$$


This gives an imbedding of hyperfinite factors which was the desired result of Ocneanu.

Remark 2. There is also a reverse statement which is much more difficult to prove [Oc].

Proposition 4 (Ocneanu). Trace Preserving Property and Condition (T). If the cell system satisfies the following condition (Trace preserving):

$$
\sum_{b_{2}, \alpha^{\prime}, \beta_{2}} \sum_{\alpha}
$$

then $\varphi^{(N)}$ is trace preserving, i.e. $\operatorname{Tr} \circ \varphi^{(N)}=\operatorname{Tr}$.

Proof of this result is given in the appendix.

Although of central importance in the work of Ocneanu, it is not immediate that these results are relevant for physics. The following proposition answers to this question.

Proposition 5. Assume that: We are given two graphs $\mathscr{G}_{1}$ and $\mathscr{G}_{2}$ and a symmetry between them. We are given sets of Boltzmann weights $W_{1}$ and $W_{2}$ attached to these graphs. If there exists a cell system between the two graphs such that the following condition is realized

(S)

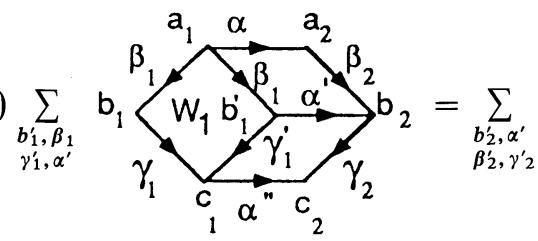

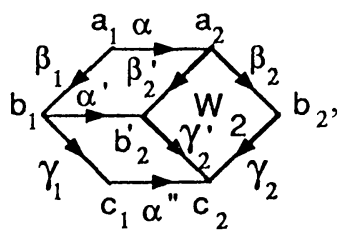

then $\varphi^{(N)}\left(U_{k}\left(\mathscr{G}_{1}\right)\right)=U_{k}\left(\mathscr{G}_{2}\right)$ for $k \leqq N$.

Proof is given in the appendix.

Consequently $\varphi^{(N)}\left(V_{1}^{(1)}\right)=V_{2}^{(1)}$ and $\varphi^{(N)}\left(V_{1}^{(2)}\right)=V_{1}^{(2)}$. We get immediately the fundamental result:

$$
Z^{\prime}\left(\mathscr{G}_{1}\right)=Z^{\prime}\left(\mathscr{G}_{2}\right)
$$

Indeed we have:

$$
\begin{aligned}
Z^{\prime}\left(\mathscr{G}_{1}\right) & =\operatorname{Tr}\left(\left(V_{1}^{(1)} V_{1}^{(2)}\right)^{M}\right)=\operatorname{Tr}\left(\varphi^{(N)}\left(\left(V_{1}^{(1)} V_{1}^{(2)}\right)^{M}\right)\right) \\
& =\operatorname{Tr}\left(\left(\varphi^{(N)}\left(V_{1}^{(1)}\right) \varphi^{(N)}\left(V_{1}^{(2)}\right)\right)^{M}\right)=\operatorname{Tr}\left(\left(V_{2}^{(1)} V_{2}^{(2)}\right)^{M}\right) \\
& =Z^{\prime}\left(\mathscr{G}_{2}\right) .
\end{aligned}
$$

This implies lots of nice things.

Suppose the first model satisfies the YBE equation, that is

$$
U_{k}\left(\mathscr{G}_{1}\right) U_{k+1}^{\prime}\left(\mathscr{G}_{1}\right) U_{k}^{\prime \prime}\left(\mathscr{G}_{1}\right)=U_{k+1}^{\prime \prime}\left(\mathscr{G}_{1}\right) U_{k}^{\prime}\left(\mathscr{G}_{1}\right) U_{k+1}\left(\mathscr{G}_{1}\right),
$$


then by applying $\varphi^{(N)}$ to this equation we get:

$$
U_{k}\left(\mathscr{G}_{2}\right) U_{k+1}^{\prime}\left(\mathscr{G}_{2}\right) U_{k}^{\prime \prime}\left(\mathscr{G}_{2}\right)=U_{k+1}^{\prime \prime}\left(\mathscr{G}_{2}\right) U_{k}^{\prime}\left(\mathscr{G}_{2}\right) U_{k+1}\left(\mathscr{G}_{2}\right),
$$

which shows that the second model is automatically integrable.

In fact we have more: the algebra underlying integrability, the smallest algebra generated by $\left\{U_{k}, k \geqq 0\right\}$ (Temperley-Lieb, Hecke, Birmann-Wenzl-Murakami [Jo2, BW, AW] for instance) is the same in both models.

From the equation,

$$
\sum_{x \in \mathscr{G}_{1}^{(0)}} Z_{x}\left(\mathscr{G}_{1}\right) S_{x}\left(\mathscr{G}_{1}\right)=\sum_{x \in \mathscr{G}_{2}^{(0)}} Z_{x}\left(\mathscr{G}_{2}\right) S_{x}\left(\mathscr{G}_{2}\right),
$$

which is interesting in itself we deduce that: the models posess the same free bulk energy. In particular after having taken the thermodynamic limit, if one model is at a second order transition point so is the other one. In this event, using the definition of the central charge as a universal correction in finite size effects, the last equation shows that both models share the same central charge.

We can now describe a general scheme for constructing new models from known ones.

1. Begin with a statistical model which possesses interesting properties and based on a graph $\mathscr{G}_{1}$.

2. Look at the symmetries of $\mathscr{G}_{1}$ and find the graphs $\mathscr{G}_{2}$ and $\mathscr{C}$.

3. Look for solutions of the system $U$ and $T$. (This is generally the difficult part.)

4. Verify that
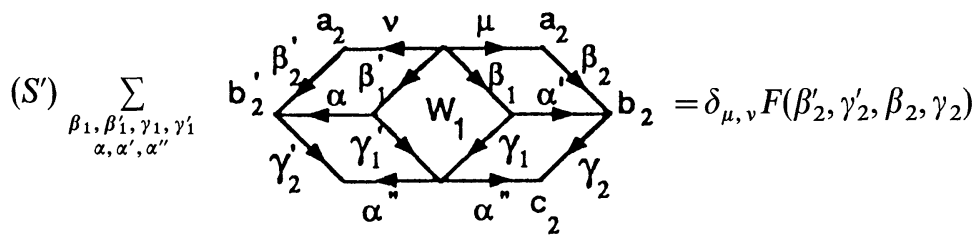

which is equivalent to: there exists $W_{2}=F\left(\beta_{2}^{\prime}, \gamma_{2}^{\prime}, \beta_{2}, \gamma_{2}\right)$ such that condition $(S)$ is satisfied.

After these steps one obtains a new model, which is the lattice analogue of orbifold construction. Condition $(S)$ means simply that the Boltzmann weights are invariant under the symmetry implemented by $\mathscr{C}$. We will explain this in a moment for ordinary symmetries associated to groups.

Remark 1. Gauge symmetry of solutions of $U$ and T. Consider a cell system

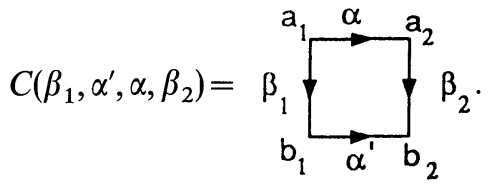

If $U\left(a_{1}, b_{1}\right)_{\beta_{1}}^{\bar{\beta}_{1}}, U\left(a_{1}, a_{2}\right)_{\alpha}^{\bar{\alpha}}, U\left(a_{2}, b_{2}\right)_{\beta_{2}}^{\bar{\beta}_{2}}$ are unitary matrices, then the family of complex numbers defined by:

$$
\begin{aligned}
& \bar{C}\left(\bar{\beta}_{1}, \bar{\alpha}^{\prime}, \bar{\alpha}, \bar{\beta}_{2}\right) \\
& =\sum_{\beta_{1}, \alpha^{\prime}, \alpha, \beta_{2}}\left(U\left(a_{1}, b_{1}\right)_{\beta_{1}}^{\beta_{1} *}\left(U\left(b_{1}, b_{2}\right)\right)_{\alpha^{\prime}}^{\prime}\right)^{*} C\left(\beta_{1}, \alpha^{\prime}, \alpha, \beta_{2}\right) U\left(a_{1}, a_{2}\right)_{\alpha}^{\bar{\alpha}} U\left(a_{2}, b_{2}\right)_{\beta_{2}}^{\bar{\beta}_{2}},
\end{aligned}
$$


is also a cell system. We will say that $C$ and $\bar{C}$ are related by a gauge transformation, which is the language of physicists, Ocneanu prefers the term perturbation. All solutions of $U$ and $T$ we give are unique up to a perturbation.

Remark 2. If $S$ is an eigenvector of $G_{1}$ such that all its components are non-zero, it is possible to define a non-positive trace on $\mathscr{A}^{N}$ using formula 2. If there exists a cell system such that $(U, T, S)$ are verified, we obtain a new equality (5), with $S_{x}\left(\mathscr{G}_{1}\right)$ and $S_{x}\left(\mathscr{G}_{2}\right)$ non-positive.

An Explicit Example. This example in the case $\mathscr{G}_{1}=A_{5}$ and at the critical point has been already considered by Pasquier [Pa.3].

Consider ABF models [ABF]. These are integrable IRF models associated to the Dynkin diagram $\mathscr{G}_{1}=A_{n}$. Parametrisation of weights are elliptic and up to a normalization factor:

$$
\begin{aligned}
& W_{1}(l-1, l, l+1, l)=W_{1}(l+1, l, l-1, l)=\alpha_{l}=h(2 \eta-u), \\
& W_{1}(l, l+1, l, l-1)=W_{1}(l, l-1, l, l+1)=\beta_{l}=h(u)\left(h\left(w_{l-1}\right) h\left(w_{l+1}\right)\right)^{1 / 2} / h\left(w_{l}\right), \\
& W_{1}(l, l+1, l, l+1)=\gamma_{l}=h(2 \eta) h\left(w_{l}+u\right) / h\left(w_{l}\right), \\
& W_{1}(l, l-1, l, l-1)=\delta_{l}=h(2 \eta) h\left(w_{l}-u\right) / h\left(w_{l}\right),
\end{aligned}
$$

where $h(u)=\theta_{1}(u) \theta_{4}(u)$ and $\theta_{1}, \theta_{4}$ are the usual Jacobi theta functions of nome $p \in]-1,1\left[, l\right.$ is an element of $\{1, \ldots, n\}$ and $w_{l}=2 l \eta$, where $\eta=\frac{\pi}{2(n+1)}$.

$p$ measures deviation from the critical point which corresponds to $p=0$ and $u$ is the spectral parameter, complex in general. Boltzmann weights can be made positive by a gauge transformation [ABF] when $u \in]-2 \eta, 2 \eta[$.

The graph $A_{n}$ posesses an obvious $Z_{2}$ symmetry: Reflection $\sigma$ around the midpoint $(\sigma(l)=n+1-l)$ leaves the graph invariant. It will be convenient to define $T=\sigma(l)$ (Fig. 13). The structure of the graph $\mathscr{G}_{2}$ depends on the parity of $n$ : when $n$ is odd it is the Dynkin diagram $D_{\frac{n+3}{2}}$, and when $n$ is even it is the Dynkin diagram

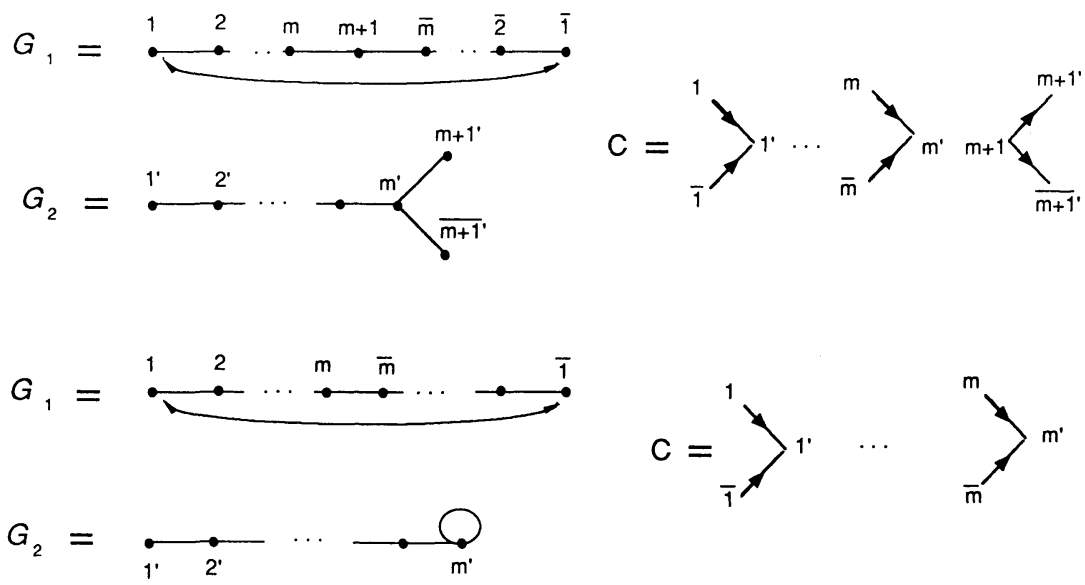

Fig. 13 
$A_{\frac{n}{2}}$ with a loop attached to one end (Fig. 13). The cell system is easily found in both cases, and we have:

- when $n=2 m+1$ odd

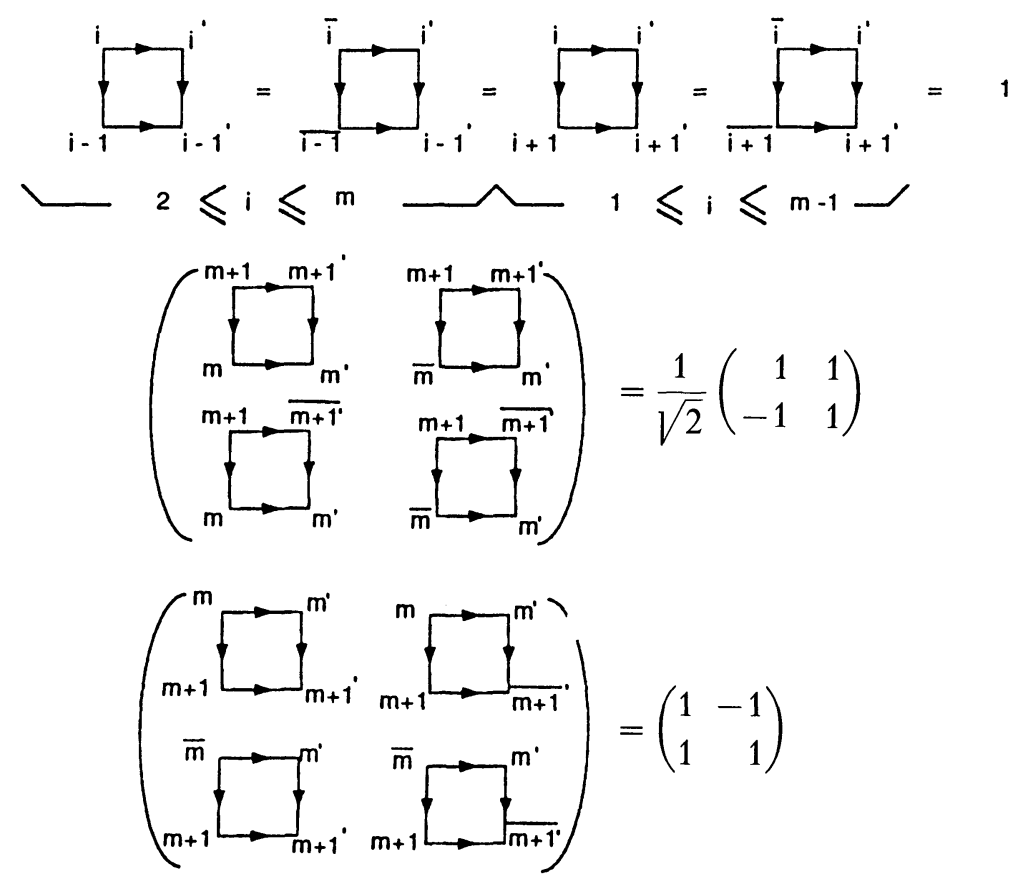

- when $n=2 m$
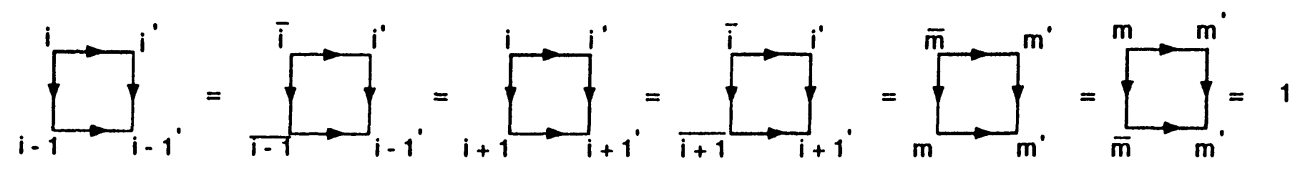

$$
2 \leqslant i \leqslant m \quad 1 \leqslant i \leqslant m-1
$$

Condition $\left(S^{\prime}\right)$ means that weights are invariant under the $Z_{2}$ symmetry, more precisely:

- when $n=2 m+1$ :

$$
\begin{aligned}
& W_{1}(i, j, k, l)=W_{1}(\bar{i}, \bar{j}, \bar{k}, \bar{l}) \quad \text { for } \quad i \notin\{m, \bar{m}, m+1\}, \\
& W_{1}(m, m-1, m-2, m-1)=W_{1}(\bar{m}, \overline{m-1}, \overline{m-2}, \overline{m-1}), \\
& W_{1}(m, m-1, m, m-1)=W_{1}(\bar{m}, \overline{m-1}, \bar{m}, \overline{m-1}), \\
& W_{1}(m, m-1, m, m+1)=W_{1}(\bar{m}, \overline{m-1}, \bar{m}, \overline{m+1}), \\
& W_{1}(m, m+1, m, m-1)=W_{1}(m, m+1, \bar{m}, \overline{m-1}), \\
& W_{1}(m, m+1, m, m+1)+W_{1}(m, m+1, \bar{m}, m+1) \\
& \quad=W_{1}(\bar{m}, m+1, \bar{m}, m+1)+W_{1}(\bar{m}, m+1, m, m+1) .
\end{aligned}
$$

In particular if $W_{1}(i, j, k, l)=W_{1}(\bar{i}, \bar{j}, \bar{k}, l)$ for every $i, j, k, l$ then the previous conditions are satisfied. 
- when $n=2 m W_{1}(i, j, k, l)=W_{1}(\bar{i}, \bar{j}, \bar{k}, \bar{l})$ : These last conditions are easily checked using the explicit parametrisation of weights. Using equation $(S)$, when $n$ is odd, we obtain a new integrable model based on $D_{n}$, this model has been constructed by Pasquier using direct calculations [Pa.4]. We give here the parametrisation of weights:

$$
\begin{aligned}
W_{2}\left(i^{\prime}, j^{\prime}, k^{\prime}, l^{\prime}\right)=W_{1}(i, j, k, l) & =W_{1}(\bar{i}, \bar{j}, \bar{k}, \bar{l}) \text { when } i^{\prime} \notin\left\{m^{\prime}, m+1^{\prime}, \overline{m+1} 1^{\prime}\right\}, \\
W_{2}\left(m^{\prime}, m-1^{\prime}, m-2^{\prime}, m-1^{\prime}\right)=W_{1}(m, m-1, m-2, m-1), & \\
W_{2}\left(m^{\prime}, m-1^{\prime}, m^{\prime}, m-1^{\prime}\right) & =W_{1}(m, m-1, m, m-1), \\
W_{2}\left(m^{\prime}, m-1^{\prime}, m^{\prime}, m+1^{\prime}\right) & =W_{2}\left(m^{\prime}, m-1^{\prime}, m^{\prime}, \overline{m+1^{\prime}}\right) \\
& =\frac{1}{\sqrt{2}} W_{1}(m, m-1, m, m+1), \\
W_{2}\left(m^{\prime}, m+1^{\prime}, m^{\prime}, m+1^{\prime}\right) & =W_{2}\left(m^{\prime}, \overline{m+1}{ }^{\prime}, m^{\prime}, \overline{m+1}{ }^{\prime}\right) \\
& =\frac{1}{2}\left(W_{1}(m, m+1, m, m+1)+W_{1}(m, m+1, \bar{m}, m+1)\right), \\
\left.W_{2}\left(m^{\prime}, m+1^{\prime}, m^{\prime}, \overline{m+1}\right)^{\prime}\right) & =W_{2}\left(m^{\prime}, \overline{m+1}{ }^{\prime}, m^{\prime}, m+1^{\prime}\right) \\
& =\frac{1}{2}\left(W_{1}(m, m+1, m, m+1)-W_{1}(m, m+1, \bar{m}, m+1)\right), \\
W_{2}\left(m+1^{\prime}, m^{\prime}, m+1^{\prime}, m^{\prime}\right) & =W_{2}\left(\overline{m+1}{ }^{\prime}, m^{\prime}, \overline{m+1}{ }^{\prime}, m^{\prime}\right) \\
& =W_{1}(m+1, m, m+1, m)+W_{1}(m+1, \bar{m}, m+1, m), \\
W_{2}\left(m+1^{\prime}, m^{\prime}, \overline{m+1}, m^{\prime}\right) & =W_{2}\left(\overline{m+1^{\prime}}, m^{\prime}, m+1^{\prime}, m^{\prime}\right) \\
& =W_{1}(m+1, m, m+1, m)-W_{1}(m+1, \bar{m}, m+1, m) .
\end{aligned}
$$

This shows the power of the method: parametrisation of weights is almost straightforwardly found compared to the direct method. Similar calculations using a different point of view have been done in [FG].

When $n$ is even, parametrisation of weights are given by:

$W_{2}\left(i^{\prime}, j^{\prime}, k^{\prime}, l^{\prime}\right)=W_{1}(i, j, k, l)$ for every $i, j, k, l$ such that if $(i, j, k, l)$ is admissible so $\left(i^{\prime}, j^{\prime}, k^{\prime}, l^{\prime}\right)$ should be chosen. Using the cell system, it is easy to convince oneself that the partition function of the model $A_{n}$ on the torus is equal to twice the partition function of the model based on the graph $\mathscr{G}_{2}$. Hence we have not constructed a new model at all, it is just the same model but associated to a different graph.

\section{Vertex-IRF Transformation and Group Theory}

It would be interesting to obtain general results on existence of solutions of $U$ and $T$. Because this is for the present time out of reach, we will rather concentrate on an important class of solutions, namely those associated to group theory. (For solutions associated to $\mathscr{U} S U(n)_{q}$ see $[\mathrm{Pa} 2]$.)

Let $G$ be a finite group and $\left(V_{i}, \pi_{i}\right)_{i=1, \ldots, m}$ its irreducible representations $\left(V_{i}\right.$ is the vector space on which $\pi_{i}$ acts). It will be convenient to think $V_{i}$ as a $G$-module. In the following we will always assume that if $(V, \pi)$ is a representation of $G, V$ is endowed with an hermitian scalar product for which $\pi$ is unitary.

Let $(V, \pi)$ be a fixed representation of $G$ (not necessarily irreducible) and $B$ an orthonormal basis of $V$. 
Fig. 14
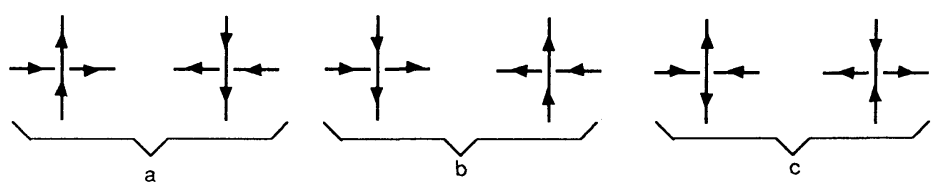

Vertex Representation. Let $R(u) \in \operatorname{End}(V \otimes V)$ be a solution of YBE equation, i.e.:

$$
(R(u) \otimes 1)\left(1 \otimes R\left(u^{\prime}\right)\right)\left(R\left(u^{\prime \prime}\right) \otimes 1\right)=\left(1 \otimes R\left(u^{\prime \prime}\right)\right)\left(R\left(u^{\prime}\right) \otimes 1\right)(1 \otimes R(u)) .
$$

We associate to this solution a vertex model where states of edges are vectors taken in a fixed basis of $V$. We define:

$$
\left\langle\beta_{1} \otimes \beta_{2}|R| \alpha_{1} \otimes \alpha_{2}\right\rangle=\sum_{\beta_{2}}^{\beta_{1}}
$$

We will assume that the model has a $G$ invariance:

$$
[R(u), \pi(g) \otimes \pi(g)]=0, \quad \forall g \in G .
$$

An important example is the 6-Vertex model which exhibits $S U(2)$ symmetry for $\Delta=\frac{a^{2}+b^{2}-c^{2}}{2 a b}=-1$ (Fig. 14). At this point the 6-Vertex model describes the WZW-SU(2) level 1 CFT.

Cell System. As a $G$-module we have:

$$
V_{i} \otimes V=\bigoplus_{j=1}^{N} \operatorname{Hom}_{G}\left(V_{j}, V_{i} \otimes V\right) \otimes V_{j},
$$

where $\operatorname{Hom}_{G}\left(V_{j}, V_{i} \otimes V\right)$ is the space of intertwiners between $\pi_{j}$ and $\pi_{i} \otimes \pi$. Let $e_{i}^{(p)}$, $p=1 \ldots \operatorname{dim}\left(V_{i}\right)$ be an orthonormal basis of $V_{i}$, and $\varphi_{i j}^{(p)}$, $\mathrm{p}=1 \ldots \operatorname{dim} \operatorname{Hom}_{G}\left(V_{j}, V_{i} \otimes V\right)$ a basis of $\operatorname{Hom}_{G}\left(V_{j}, V_{i} \otimes V\right)$ such that $\left(\varphi_{i j}^{(p)}\left(e_{j}^{(q)}\right)\right)_{p, q, j}$ is an orthonormal basis of $V_{i} \otimes V$.

We define:

$$
\left\langle\varphi_{i j}^{(p)}\left(e_{j}^{(q)}\right) \mid e_{i}^{(r)} \otimes \beta\right\rangle=P_{j}^{\longrightarrow} \beta .
$$

This system of complex numbers is a cell system. This system satisfies the relations $U_{1}$ and $T_{1}$ :

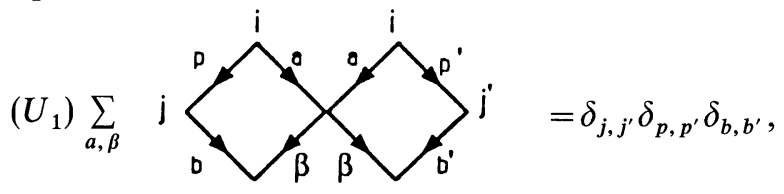

$\left(T_{1}\right) \sum_{\beta, b}$

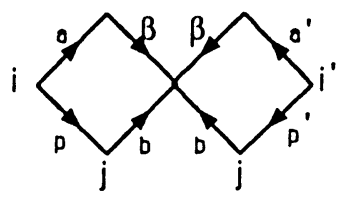

$=\delta_{i, i^{\prime}} \delta_{a, a^{\prime}} \delta_{p, p^{\prime}} \frac{\operatorname{dim} \pi_{j}}{\operatorname{dim} \pi_{i}}$ 
Proof of these relations are given in the appendix. At this stage we can work out the simplest example, namely $G=Z_{n}$. Let $\omega=e^{2 i \pi / n}$. There are $n$ irreducible onedimensional representations $\left(\pi_{i}\right)_{i=1, \ldots, n}$, defined by:

$$
\pi_{i}(k)=\omega^{k i}, \quad \forall k \in G .
$$

Let us define the 2-dim representation $\pi=\pi_{1} \otimes \pi_{-1}$. We have $\pi_{i} \otimes \pi=\pi_{i-1} \oplus \pi_{i+1}$. Let $(+,-)$ be a basis of $V$ adapted to this decomposition. An immediate calculation shows that:
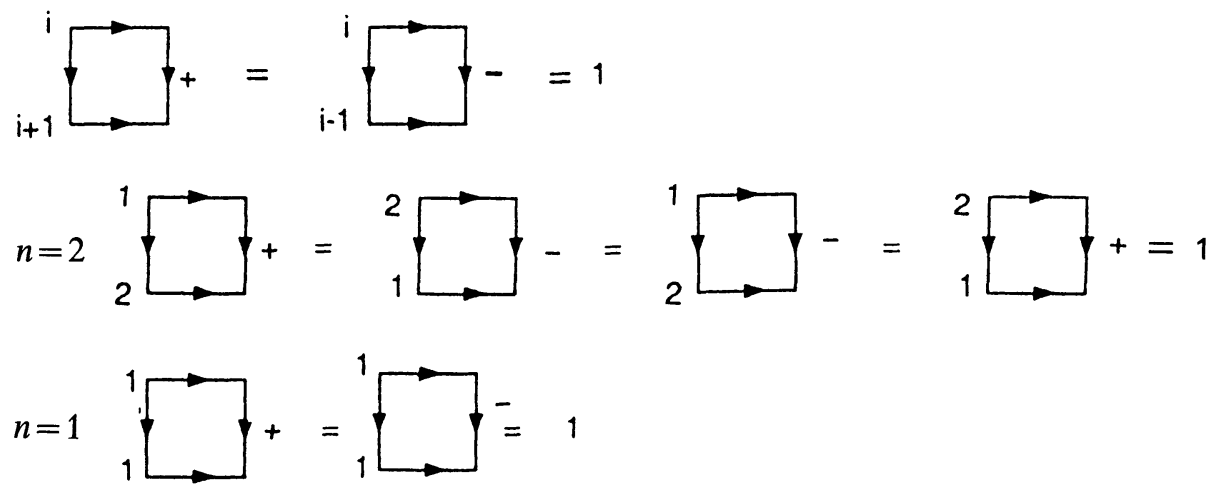

the other cells are zero. Other cell systems associated to non-abelian groups will be used later.

IRF Representation. Let $j_{V}: V \rightarrow V^{*}$ be the antilinear isomorphism induced by the hermitian product.

Lemma. If $\left(V_{1}, \pi_{1}\right)$ and $\left(V_{2}, \pi_{2}\right)$ are irreducible representations of $G$, if $(W, \varrho)$ is a representation of $G$ and $\varphi: V_{1} \rightarrow W$ and $\psi: V_{2} \rightarrow W$ are intertwiners then $j_{V_{2}}^{-1} \circ \psi^{t} \circ j_{W} \circ \varphi$ is an intertwiner denoted $(\varphi, \psi)$.

As a result:

- if $\pi_{1}$ and $\pi_{2}$ are non-equivalent then $(\varphi, \psi)=0$,

- if $\pi_{1}=\pi_{2}$ then $(\varphi, \psi)=\lambda 1_{V_{1}}$. In that case, we will replace $(\varphi, \psi)$ by the pure number $\lambda$. If $x$ is a unit vector in $V_{1},(\varphi, \psi)=\langle\psi(x) \mid \varphi(x)\rangle$.

We have:

$$
\begin{aligned}
\left(V_{i} \otimes V\right) \otimes V & =\left(\underset{j}{\oplus} \operatorname{Hom}_{G}\left(V_{j}, V_{i} \otimes V\right) \otimes V_{j}\right) \otimes V \\
& =\bigoplus_{j, k} \operatorname{Hom}_{G}\left(V_{j}, V_{i} \otimes V\right) \otimes \operatorname{Hom}_{G}\left(V_{k}, V_{j} \otimes V\right) \otimes V_{k} .
\end{aligned}
$$

Define

$$
\varphi=\left(\varphi_{i j}^{\left(p_{1}\right)} \otimes 1_{V}\right) \varphi_{k j}^{\left(p_{2}\right)} \in \operatorname{Hom}_{G}\left(V_{k}, V_{i} \otimes V \otimes V\right)
$$

and

$$
\psi=\left(\varphi_{i j^{\prime}}^{\left(p_{1}^{\prime}\right)} \otimes 1_{V}\right) \varphi_{l j^{\prime}}^{\left(p_{2}^{\prime}\right)} \in \operatorname{Hom}_{G}\left(V_{l}, V_{i} \otimes V \otimes V\right) .
$$

It is easy to verify that $\left(1_{V_{\imath}} \otimes R\right)$ is element of $\operatorname{Hom}_{G}\left(V_{i} \otimes V \otimes V, V_{i} \otimes V \otimes V\right)$. 
It follows from the lemma that $\left(\psi,\left(1_{V_{i}} \otimes R\right) \varphi\right)=0$ if $k \neq l$, and is a $\mathbb{C}$ number when $k=l$. It is convenient to use the following graphical notation:

$$
\left(\psi,\left(1_{V_{i}} \otimes R\right) \varphi\right)={ }_{\mathrm{K}}
$$

The relation between Vertex representation and IRF representation can be written:

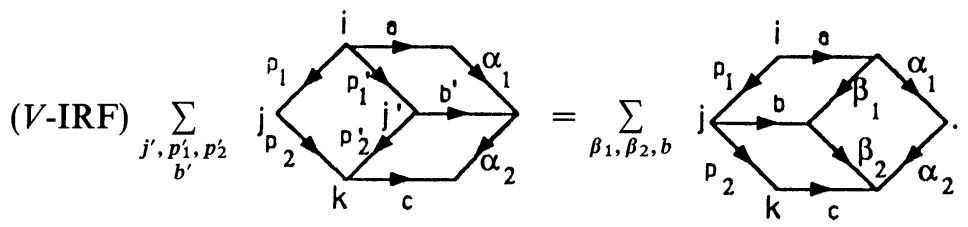

Proof of this relation is given in the appendix. Using relation $\left(U_{1}\right)$ this last equation permits us to recover Vertex representation of $R$ from IRF representation of $R$ and conversely.

Let $\mathscr{G}_{2}$ be the graph with one node and $n$ arrows. Define a matrix $\left(N(\pi)_{i j}\right)$ by:

$$
\pi_{i} \otimes \pi=\bigoplus_{j} N(\pi)_{i j} \pi_{j}
$$

Let $\mathscr{G}_{1}$ be the graph having $N(\pi)$ for incidence matrix. Let $\chi_{\pi}$ be the character of the representation $\pi$. We have $\chi_{\pi_{i}}(g) \chi_{\pi}(g)=\sum_{j} N(\pi)_{i j} \chi_{\pi_{j}}(g)$, where $g \in G$. This shows that the eigenvalue of $N(\pi)$ are $\chi_{\pi}(\gamma)=\beta_{\gamma}$, where $\gamma$ is a conjugacy class of the group $G$, and the eigenvector associated to $\beta_{\gamma}$ is $\left(\chi_{\pi_{i}}(\gamma)\right)_{i=1, \ldots, m}$. We always have $\left|\chi_{\pi}(\gamma)\right|$ $\leqq \chi_{\pi}(e)=\operatorname{dim} \pi$, where $e$ is the unit element of the group $G$. As a result $N(\pi)$ admits a largest eigenvalue $\beta=\operatorname{dim} \pi$ associated to the eigenvector $S=\left(\operatorname{dim} \pi_{i}\right)_{i=1, \ldots, m}$. If we introduce $C$ the $m \times 1$ matrix such that $C_{i 1}=\operatorname{dim} \pi_{i}$, then $G_{1} C=C G_{2}$ and ClebschGordan coefficients defined by (6) are a cell system between $\mathscr{G}_{1}$ and $\mathscr{G}_{2} . U_{1}$ and $T_{1}$ are equivalent to $U$ and $T$.

\section{Examples}

In this part we will discuss critical integrable $c=1$ models and give examples of $c=\frac{3}{2}$ [DSZ] models. First of all we have to remind the reader how integrable models based on quantum groups are built [Ji]. For simplicity we will restrict ourselves to the Hopf algebra $A=\mathscr{U} S U(2)_{q}$. Let $V_{j}$ be the highest weight $A$-module of dimension $2 j+1$ where $j \in \frac{1}{2} \mathbb{N}$. Let $R(x) \in \operatorname{End}\left(V_{j} \otimes V_{j}\right)$ be the trigonometric solution of YBE constructed by Jimbo. $R(x)$ is entirely characterized (up to a multiplicative constant) by the two following equations:

$$
\begin{aligned}
{[R(x), \Delta(a)] } & =0, \quad \forall a \in A, \\
R(x)\left(x J_{-} \otimes q^{h / 2}+q^{-h / 2} \otimes J_{-}\right) & =\left(J_{-} \otimes q^{h / 2}+x q^{-h / 2} \otimes J_{-}\right) R(x) .
\end{aligned}
$$


$A$ acts via $\Delta$ on $V_{j} \otimes V_{j}$ as the $[j] \otimes[j]$ representation. From $[j] \otimes[j]=\bigoplus_{k=0}^{2 j}[k]$ and Eq. (7) we deduce $R(x)=\sum_{k=0}^{2 j} a_{k}(x) P^{(k)}$, where $P^{(k)}$ is the projection on the irreducible $A$-module of dimension $2 k+1$. Equation (8) implies that:

$$
\frac{a_{k-1}(x)}{a_{k}(x)}=\frac{x-q^{2 k}}{1-x q^{2 k}}
$$

It remains to calculate $P^{(k)}$ in an explicit basis to obtain an explicit description of $R(x)$ (for $j=\frac{1}{2}$ and $j=1$, see [Pa.2]).

$j=\frac{1}{2}$ Case. In this case

$$
R(x)=\left(1-x q^{2}\right) P^{(1)}+\left(x-q^{2}\right) P^{(0)}=1-x q^{2}+(x-1)\left(1+q^{2}\right) P^{(0)} .
$$

Statistical models are defined by face operators $U_{k}(x)=1^{\otimes^{k}} \otimes R(x) \otimes 1^{\otimes N-k-1}$, where $N$ is the number of edges of the lattice. $e_{k}=1^{\otimes^{k}} \otimes P^{(0)} \otimes 1^{\otimes{ }^{N-k-1}}$ define a representation of the Temperley-Lieb algebra. In a basis $(+,-)$ in which $h=\left(\begin{array}{cc}1 / 2 & 0 \\ 0 & -1 / 2\end{array}\right)$, non-zero elements of $P^{(0)}$ are:
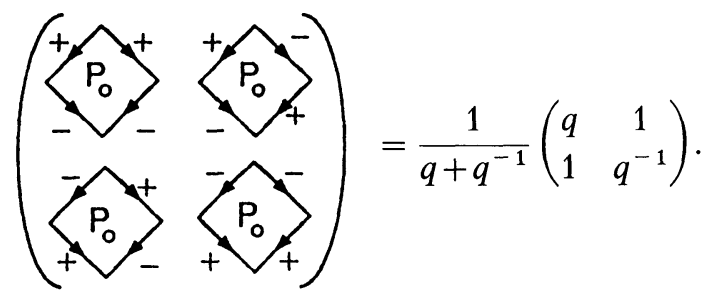

From $[R(x), \Delta(h)]=0$, we obtain $\left[R(x), \Delta\left(\exp \left(\frac{i 4 \pi k h}{n}\right)\right)\right]=0$. Because $\Delta\left(\exp \left(\frac{i 4 \pi k h}{n}\right)\right)=\pi(g) \otimes \pi(g)$, where $g=\omega^{k}$ and $\pi=\pi_{1} \oplus \pi_{-1}$ we can apply a Vertex-IRF transformation using Clebsh-Gordan coefficients of the group $Z_{n}$. The graph associated to the IRF model is the Dynkin diagram of the affine algebra $A_{n-1}^{(1)}$ (when $n=1$ the graph has only one point and two arrows: we recover the $6 \mathrm{~V}$ model). The non-zero matrix element of $P^{(0)}$ in this representation are:

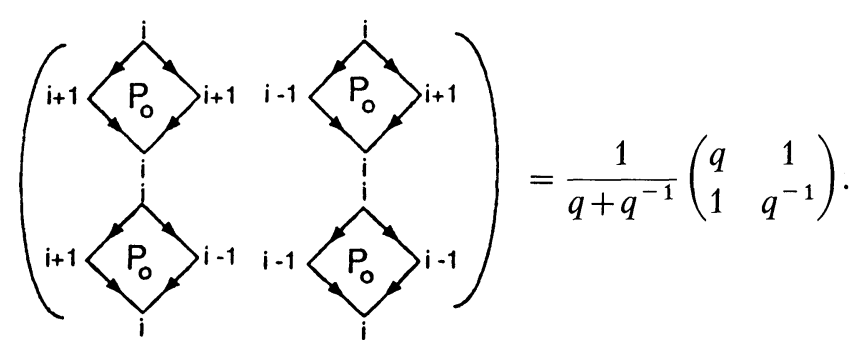

Models associated to this representation of the Temperley-Lieb algebra are models called " $Z_{f}$ " by Pasquier. His parameter $z$ is equal to $q^{2}$. The $6 \mathrm{~V}$ model in the regime $|\Delta| \leqq 1$ renormalizes to a CFT consisting of a free compactified real 
boson on $S^{1}$ with radius $r$ defined by $-\cos \left(\frac{\pi r^{2}}{2}\right)=\frac{q+q^{-1}}{2}$. These " $Z_{f}$ " models correspond in the language of CFT to constructing the $Z_{n}$ orbifolds.

When $q= \pm 1 A$ degenerates to $\mathscr{U} S U(2)$, Eq. (7) reduces to $[R(x), g \otimes g]=0$ $\forall g \in S U(2)$. As a result this equation is valid for every $g$ in $G$ where $G$ is a finite subgroup $S U(2)$. Finite subgroups of $S U(2)$ are known, they are the inverse image in the mapping $S U(2) \rightarrow S O(3)$ of the celebrated crystallographics groups. Mac Kay has found a one to one mapping between these binary groups and Dynkin diagrams of simply laced affine algebra without torsion [McK]. Let $\pi$ be the two-dimensional representation (called natural representation) of the binary group $G$ coming from the inclusion $G \subset S U(2)$. $N(\pi)$ is the incidence matrix of an extended Dynkin diagram, and this is a one to one mapping. IRF models constructed via the Vertex-IRF correspondence applied to the $6 \mathrm{~V}$ by modding out by a subgroup $G$ gives all models constructed by Pasquier with extended Dynkin diagrams. These models describe the orbifolds (WZW $S U(2)-l e v e l 1) / G$ [Gi.2, $\mathrm{Pa} .5]$. This shows in this particular case that Vertex-IRF correspondence is the lattice analogue of the construction of orbifold models. Equality of the central charge of Vertex model and of IRF models in general is an indication of this conjecture. Face operators of the 6-Vertex model are $R_{k}(u)=1+u e_{k}$, where $u$ is a positive anisotropy parameter. Let $R_{k}^{\prime}(u)$ be the face operators of the system after a rotation of $\frac{\pi}{2}$. We have $R_{k}^{\prime}(u)=\frac{u}{2} R_{k}\left(\frac{4}{u}\right)$, which shows that the isotropic point is obtained at $u=2$. Let $W\left(i, j^{\prime}, k, j\right)(u)$ be the Boltzmann weights of the IRF model obtained by the mapping $6 V$-IRF using a cell system associated to the group $G$ and its two-dimensional representation. We must have

$$
W\left(i, j^{\prime}, k, j\right)(u)=\frac{u}{2} W\left(j, i, j^{\prime}, k\right)\left(\frac{4}{u}\right) \frac{f(j) f\left(j^{\prime}\right)}{f(i) f(k)} .
$$

We obtain that:

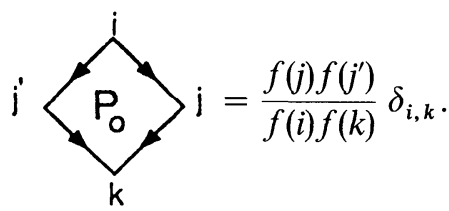

From $e_{k}^{2}=e_{k}$ we deduce that $\sum_{j} N_{i j} f(j)^{2}=2 f(i)^{2}$, which implies $f(i)=\left(\operatorname{dim} \pi_{i}\right)^{1 / 2}$. This proof does not use at all properties of cell systems. We can give here another proof which uses only property $U$ and $T$. If we introduce $F_{c c^{\prime}}^{\varepsilon f^{\prime}}\left(i, j^{\prime}, k ; k^{\prime}, j\right)$ defined by:

$$
F_{c c^{\prime}}^{\varepsilon \varepsilon^{\prime}}\left(i, j^{\prime}, k ; k^{\prime}, j\right)=\sum_{a, b, b^{\prime}} j_{c}^{\prime}
$$

where $\varepsilon, \varepsilon^{\prime}$ are elements of $(+,-)$ and $\mp=-$ and $==+$, we obtain:

$$
W\left(i, j^{\prime}, k, j\right)=\delta_{j, j^{\prime}}+\frac{u}{2} \sum_{\varepsilon, \varepsilon^{\prime}} F_{c c}^{\varepsilon, \varepsilon^{\prime}}\left(i, j^{\prime}, k ; k, l\right)
$$


( $c$ is any fixed element of the basis of $V_{k}$ ). Equation (9) implies then

$$
\sum_{\varepsilon, \varepsilon^{\prime}} F_{c c}^{\varepsilon, \varepsilon^{\prime}}\left(i, j^{\prime}, k ; k, j\right)=\frac{f(j) f\left(j^{\prime}\right)}{f(i) f(k)} \delta_{j, j^{\prime}}
$$

It remains to calculate $f$. From positivity of Boltzmann weights it is sufficient to calculate the square module of $F\left(i, j^{\prime}, k, j\right)=\sum_{\varepsilon, \varepsilon^{\prime}} F_{c c}^{\varepsilon, \varepsilon^{\prime}}\left(i, j^{\prime}, k, k, j\right)$.

From $\sum_{\varepsilon, \varepsilon^{\prime}} F_{c c^{\prime}}^{\varepsilon, \varepsilon^{\prime}}\left(i, j^{\prime}, k ; k^{\prime}, j\right)=\delta_{k, k^{\prime}} \delta_{c, c^{\prime}} F^{\varepsilon, \varepsilon^{\prime}}\left(i, j^{\prime}, k ; k, j\right)$, we have

$$
\left|F\left(i, j^{\prime}, k, j\right)\right|^{2}=\frac{1}{\operatorname{dim} \pi_{k}} \sum_{c, c^{\prime}}\left|\sum_{\varepsilon, \varepsilon^{\prime}} F_{c, c^{\prime}}^{\varepsilon, \varepsilon^{\prime}}\left(i, j^{\prime}, k ; k, j\right)\right|^{2} .
$$

Hence

$$
\operatorname{dim} \pi_{i}\left|F\left(i, j^{\prime}, k, j\right)\right|^{2}=\sum_{k, k^{\prime}, c, c^{\prime}}\left|\sum_{\varepsilon, \varepsilon^{\prime}} F_{c, c^{\prime}}^{\varepsilon, \varepsilon^{\prime}}\left(i, j^{\prime}, k ; k^{\prime}, j\right)\right|^{2}
$$

[because $F\left(i, j^{\prime}, k, j\right)$ is proportional to $\delta_{i, k}$ ]

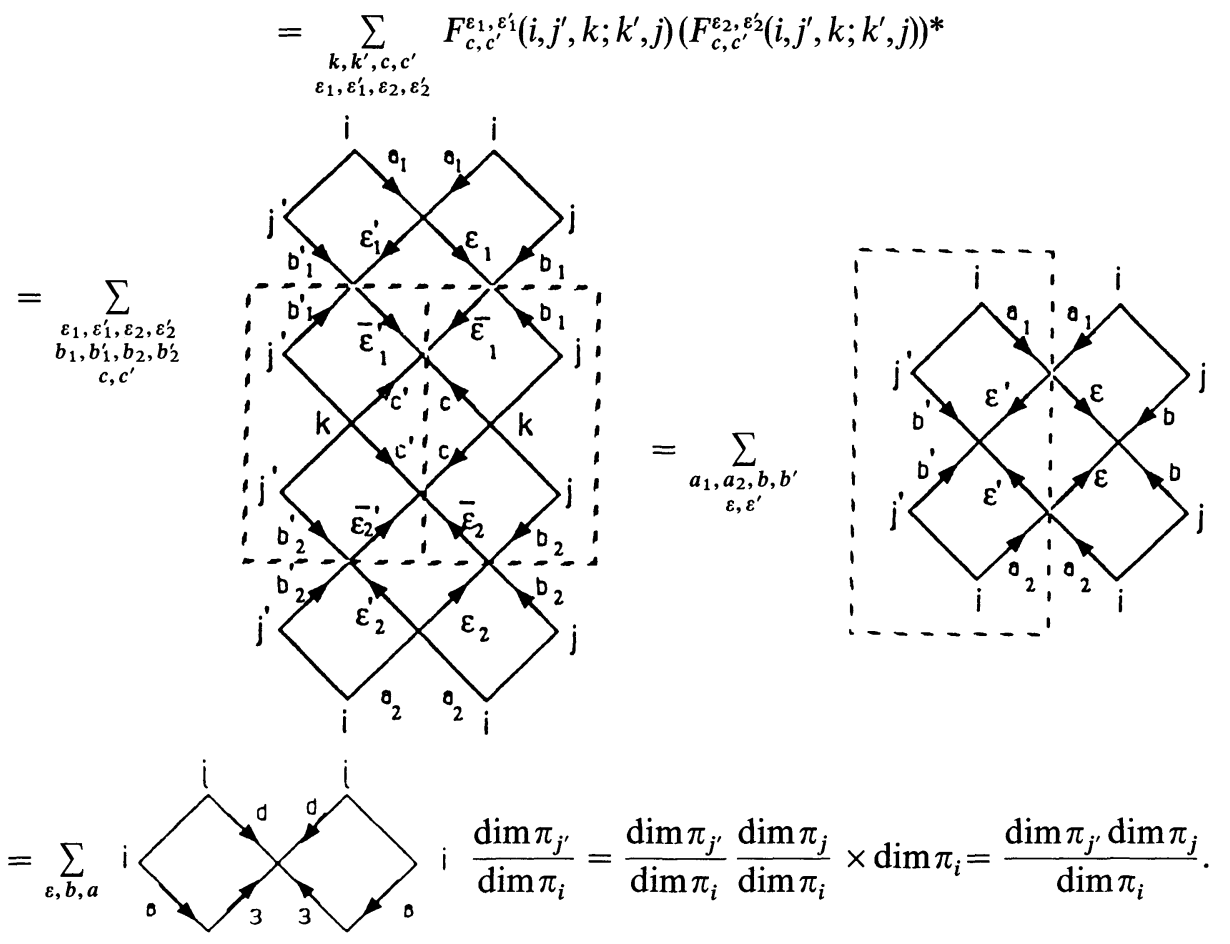

As a result $F\left(i, j^{\prime}, k, j\right)=\delta_{i, k} \frac{\left(S_{j} S_{j^{\prime}}\right)^{1 / 2}}{S_{i}}$ which is the well known result.

$j=1$ Case. In this case expression of $R(x)$ reads as follows:

$$
R(x)=\left(1-x q^{4}\right)\left(1-x q^{2}\right) P^{(2)}+\left(x-q^{4}\right)\left(1-x q^{2}\right) P^{(1)}+\left(x-q^{2}\right)\left(x-q^{4}\right) P^{(0)} .
$$



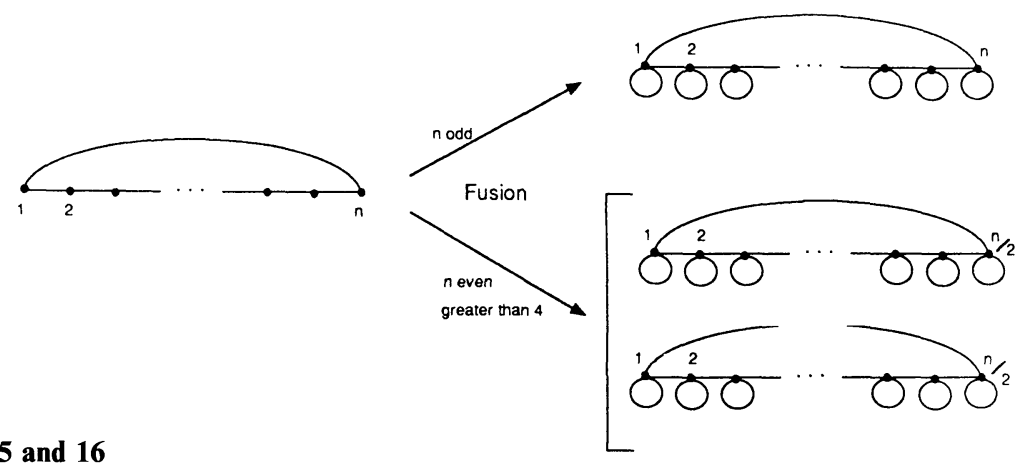

Figs. 15 and 16

$R(x)$ defines a vertex model known as $19 V$ [because $R(x)$ has 19 non-zero matrix elements]. This model is critical for $|q|=1$ and it is believed to renormalize to a SUSY $N=1$ CFT [DSZ]. When $q=1$ it describes the WZW SU(2)-level 2 CFT. Using the analysis of [DGH], we deduce that the $19 \mathrm{~V}$ renormalizes to a point on the line of the moduli space of SUSY $N=1$ CFT containing the point WZW SU(2)-level 2. This is the line $s-a[\mathrm{DGH}]$ and the expression of the coupling constant $g$ is given in [DSZ]. Matrix elements of $P^{(0)}$ and $P^{(1)}$ have been calculated in [Pa.2]. In this part we will discuss fusion of IRF models associated to extended Dynkin Diagram. Mac Kay correspondence will help us to understand this fusion process.

Let $\varrho_{j}$ be the highest weight representation of spin $j$ of $\mathscr{U} S U(2)_{q}$. We have $[R(x)$, $\left.\left(\varrho_{1} \otimes \varrho_{1}\right) \Delta(a)\right]=0 \quad \forall a \in \mathscr{U} S U(2)_{q} . \quad$ Let $\quad g=\exp \left(\frac{i 4 \pi k h}{n}\right), \quad$ from $\quad\left(\varrho_{1} \otimes \varrho_{1}\right) \Delta(g)$ $=\pi(g) \otimes \pi(g)$, where $\pi=\pi_{2} \oplus \pi_{0} \oplus \pi_{-2}$, we can proceed as in the $\left(j=\frac{1}{2}\right)$ case. Using the $V$-IRF transformation we obtain an IRF model associated to a graph $\mathscr{G}_{2}$ we now describe:

when $n$ is odd, $\mathscr{G}_{2}$ is connected and is pictured in Fig. 15,

when $n$ is even, $\mathscr{G}_{2}$ has two connected components, each of them is pictured in Fig. 16 having each $\frac{n}{2}$ nodes.

When $q=1, \varrho_{j}$ degenerates into the representation of spin $j$ of $S U(2)$. From $\left(\varrho_{j} \otimes \varrho_{\frac{1}{2}}\right)(g)=\varrho_{j+\frac{1}{2}}(g) \oplus \varrho_{j-\frac{1}{2}}(g)$, we deduce $N\left(\varrho_{j}\right) N\left(\varrho_{\frac{1}{2}}\right)=N\left(\varrho_{j+\frac{1}{2}}\right)+N\left(\varrho_{j-\frac{1}{2}}\right)$. This recurrence equation permits us to calculate the graph associated to fused IRF models. After one fusion the graph $\mathscr{G}_{2}$ is defined by $N\left(\varrho_{1}\right)$ and are pictured in Figs. 16 and 17. Boltzmann weights of these IRF are obtained by a Vertex-IRF transformation. To calculate them we prefer to use a fusion technique [KRS], which is an inhomogeneous block spin transformation. The idea is to construct the $R^{(1)}(x)$ matrix in spin one representation from the $R^{\left(\frac{1}{2}\right)}(x)$ matrix in spin one-half representation. More precisely let us define $\bar{R}(x)$ acting on $\operatorname{End}\left(\left(V_{\frac{1}{2}}\right)^{\otimes 4}\right)$ by:

$$
\bar{R}(x)=R_{2}^{\left(\frac{1}{2}\right)}\left(x q^{-2}\right) R_{1}^{\left(\frac{1}{2}\right)}(x) R_{3}^{\left(\frac{1}{2}\right)}(x) R_{2}^{\left(\frac{1}{2}\right)}\left(x q^{2}\right) .
$$

$\bar{R}(x)$ is a solution of YBE. From $R\left(q^{2}\right)=\left(1-q^{4}\right) P^{(1)}$, we see that:

$$
\bar{R}(x) P_{1}^{(1)}=P_{3}^{(1)} R_{2}^{\left(\frac{1}{2}\right)}(x) R_{1}^{\left(\frac{1}{2}\right)}\left(x q^{-2}\right) R_{3}^{\left(\frac{1}{2}\right)}\left(x q^{2}\right) R_{2}^{\left(\frac{1}{2}\right)}(x) .
$$




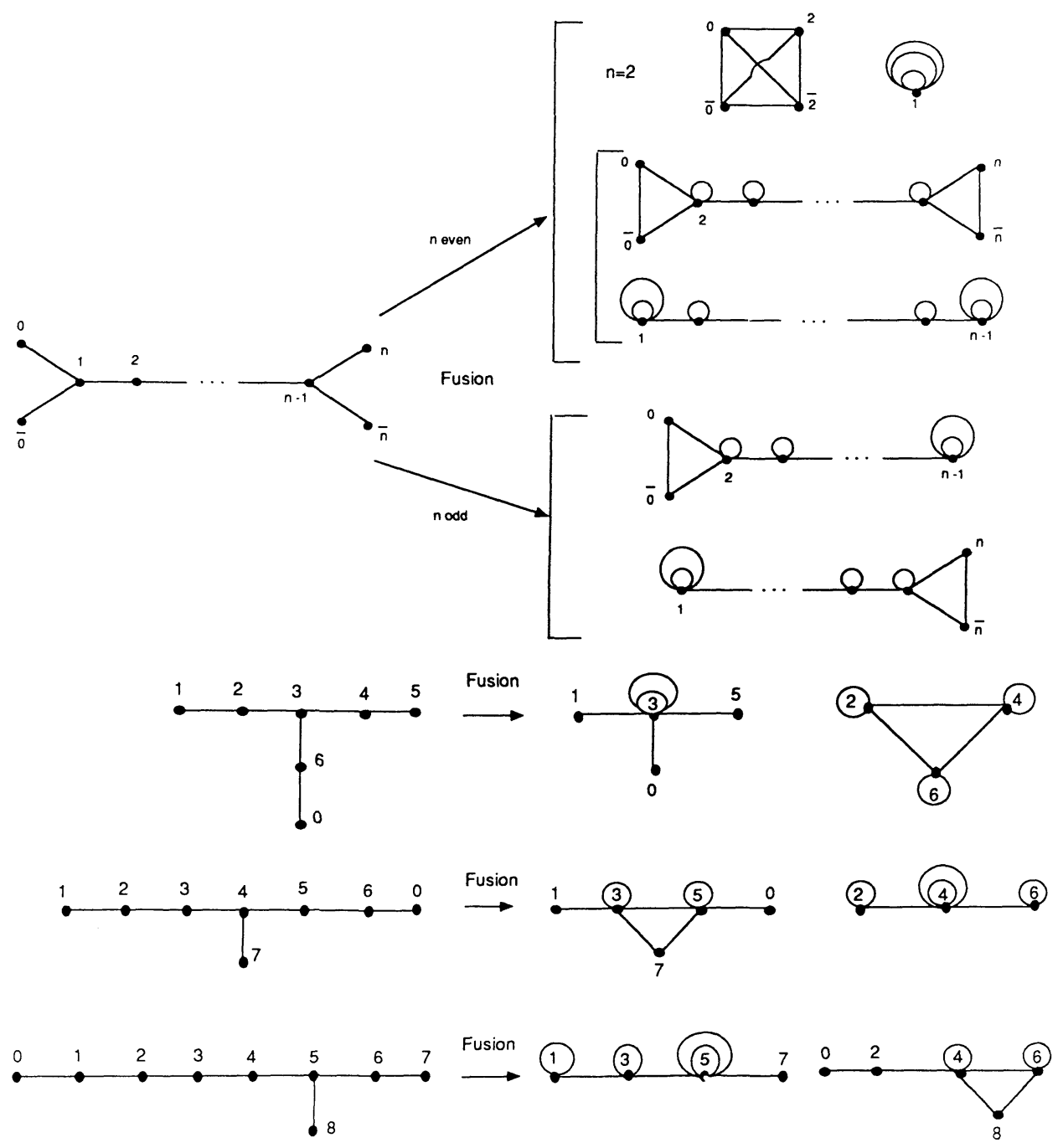

Fig. 17

This proves that we can restrict this $R$ matrix to the spin one component of $V_{\frac{1}{2}} \otimes V_{\frac{1}{2}}$. As a result, up to a multiplicative constant, we have

$$
R^{(1)}(x)=P_{1}^{(1)} P_{3}^{(1)} \bar{R}(x) P_{1}^{(1)} P_{3}^{(1)} .
$$

The limit $q$ goes to 1 is singular. If we put $q=e^{\lambda}, x=e^{\lambda u}$ and take the limit $\lambda$ goes to zero, $\frac{1}{\lambda} R^{\left(\frac{1}{2}\right)}(x)$ has the limit (up to a scalar) $1-\frac{2 u}{u+1} P^{(0)}$. Using the Vertex-IRF transformation in the expression (10) we obtain an IRF model associated to the graph $\mathscr{G}_{3}$, where heights $(i, j, k, l)$ are irreducible representations of $G$ and arrows 
$(a, b, c, d)$ are labelled by irreducible representations of $G$, such that $(i, a),(a, j),(j, b)$, $(b, k),(k, c),(c, l),(l, d),(d, a)$ are neighbours on the graph $\mathscr{G}_{1}$. The incidence matrix of $\mathscr{G}_{3}$ is equal to $N\left(\varrho_{1}\right)+1$. Although $\mathscr{G}_{3}$ differs from $\mathscr{G}_{2}$ by a loop to each point, the statistical models are equal due to the projection on the spin one component. From the definition of fusion, we have:
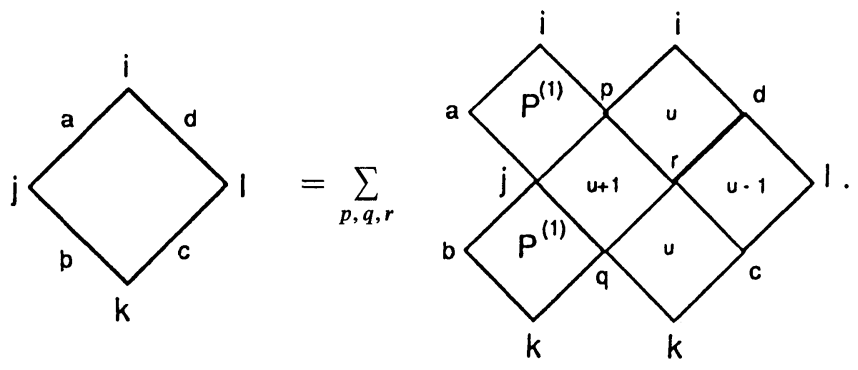

When written completely this is a sum of 64 terms. After reduction one ends with only 14 terms. Finally:

$$
\begin{aligned}
W(i, j, k, l \mid a, b, c, d) & =\delta_{j, l} \delta_{a, d} \delta_{b, c}+\frac{u(u-1)}{(u+1)(u+2)} \delta_{i, k} \delta_{a, b} \delta_{c, d} \frac{\left(S_{j} S_{l}\right)^{1 / 2}}{S_{i}} \\
& +\frac{\left(u+\frac{1}{2}\right)^{2}}{(u+1)(u+2)} \delta_{i, j} \delta_{j, k} \delta_{k, l} \frac{\left(S_{a} S_{b} S_{c} S_{d}\right)^{1 / 2}}{S_{i}^{2}}-\frac{2 u}{(u+2)} \delta_{a, b} \delta_{b, c} \delta_{c, d} \frac{\left(S_{j} S_{l}\right)^{1 / 2}}{S_{a}} \\
& -\frac{u^{2}}{(u+1)(u+2)}\left(\delta_{i, j} \delta_{j, k} \delta_{c, d} \frac{\left(S_{a} S_{b} S_{l}\right)^{1 / 2}}{S_{i}^{3 / 2}}+\delta_{i, k} \delta_{k, l} \delta_{a, b} \frac{\left(S_{d} S_{c} S_{j}\right)^{1 / 2}}{S_{i}^{3 / 2}}\right) \\
& -\frac{u+1}{u+2}\left(\delta_{i, j} \delta_{j, l} \delta_{b, c} \frac{\left(S_{a} S_{d}\right)^{1 / 2}}{S_{i}}+\delta_{j, l} \delta_{j, k} \delta_{a, d} \frac{\left(S_{b} S_{c}\right)^{1 / 2}}{S_{j}}\right) \\
& +\frac{u}{u+2}\left(\delta_{k, l} \delta_{a, b} \delta_{a, d} \frac{\left(S_{j} S_{c}\right)^{1 / 2}}{\left(S_{a} S_{k}\right)^{1 / 2}}+\delta_{j, k} \delta_{a, d} \delta_{d, c} \frac{\left(S_{b} S_{l}\right)^{1 / 2}}{\left(S_{j} S_{c}\right)^{1 / 2}}\right. \\
& \left.+\delta_{i, j} \delta_{b, c} \delta_{c, d} \frac{\left(S_{a} S_{l}\right)^{1 / 2}}{\left(S_{j} S_{c}\right)^{1 / 2}}+\delta_{i, l} \delta_{a, b} \delta_{b, c} \frac{\left(S_{j} S_{d}\right)^{1 / 2}}{\left(S_{a} S_{i}\right)^{1 / 2}}\right) \\
& -\frac{u-1}{2(u+1)}\left(\delta_{i, j} \delta_{k, l} \delta_{b, d} \frac{\left(S_{a} S_{c}\right)^{1 / 2}}{\left(S_{i} S_{k}\right)^{1 / 2}}+\delta_{i, l} \delta_{j, k} \delta_{a, c} \frac{\left(S_{b} S_{d}\right)^{1 / 2}}{\left(S_{i} S_{j}\right)^{1 / 2}}\right) .
\end{aligned}
$$

These models should describe (WZW level 2)/G conformal field theories. We hope to report elsewhere the study of these models.

An Example not Associated in Group Theory: The Cell System Connecting $A_{11}$ to $E_{6}$. In this part we prove an unexpected result: there exists a cell system connecting $A_{11}$ and $E_{6}$. Moreover, existence of this cell system proves that it is necessary to investigate cell system not connected to obvious symmetry of graphs. 

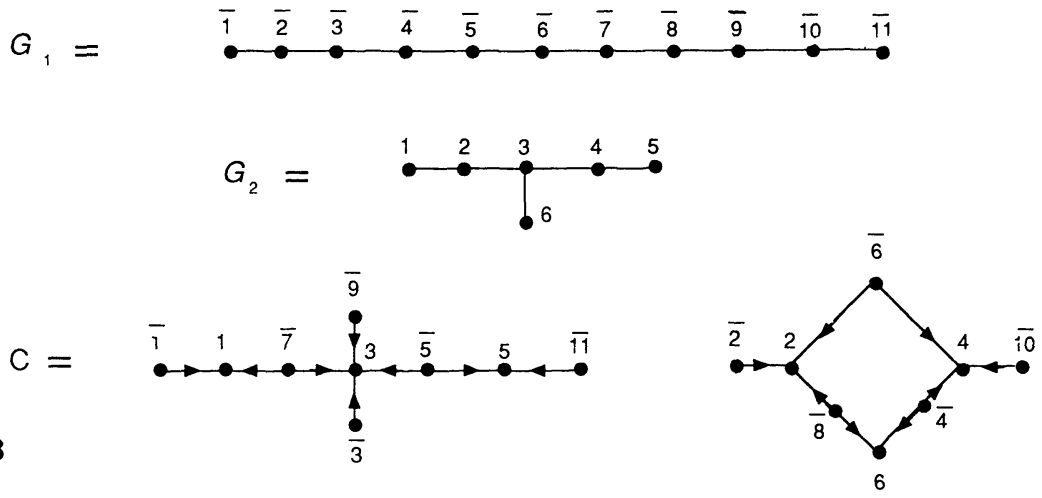

$A_{11}, E_{6}$ and matrix $C$ are pictured in Fig. 18.

We have solved equations $U$ and $T$, the solution up to a perturbation is:

$$
\begin{aligned}
& C(\overline{1}, \overline{2}, 1,2)=1 \text {, } \\
& C(\overline{2}, \overline{1}, 2,1)=C(\overline{2}, \overline{3}, 2,3)=1 \text {, } \\
& C(\overline{3}, \overline{4}, 3,4)=C(\overline{3}, \overline{2}, 3,2)=-C(\overline{3}, \overline{4}, 3,6)=1 \text {, } \\
& -C(\overline{4}, \overline{5}, 4,5)=1, C(\overline{4}, \overline{3}, 4,3)=C(\overline{4}, \overline{5}, 6,3)=3^{-1 / 4} \text {, } \\
& -C(\overline{4}, \overline{3}, 6,3)=C(\overline{4}, \overline{5}, 4,3)=\left(1-\frac{1}{\sqrt{3}}\right)^{1 / 2} \text {, } \\
& C(\overline{5}, \overline{4}, 3,6)=C(\overline{5}, \overline{6}, 3,2)=1 \text {, } \\
& -C(\overline{5}, \overline{4}, 5,4)=C(\overline{5}, \overline{6}, 3,4)=(2 \sqrt{3}-3)^{1 / 2}, \\
& C(\overline{5}, \overline{4}, 3,4)=C(\overline{5}, \overline{6}, 5,4)=\sqrt{3}-1 \text {, } \\
& C(\overline{6}, \overline{7}, 2,1)=C(\overline{6}, \overline{5}, 4,5)=1 \text {, } \\
& C(\overline{6}, \overline{5}, 2,3)=C(\overline{6}, \overline{7}, 4,3)=\frac{1}{2}(\sqrt{3}+1)^{1 / 2}, \\
& C(\overline{6}, \overline{5}, 4,3)=-C(\overline{6}, \overline{7}, 2,3)=\frac{1}{2}(3-\sqrt{3})^{1 / 2}, \\
& C(\overline{7}, \overline{6}, 3,4)=C(\overline{7}, \overline{8}, 3,6)=1 \text {, } \\
& C(\overline{7}, \overline{8}, 1,2)=-C(\overline{7}, \overline{6}, 3,2)=(2 \sqrt{3}-3)^{1 / 2}, \\
& C(\overline{7}, \overline{8}, 3,2)=C(\overline{7}, \overline{6}, 1,2)=\sqrt{3}-1 \text {, } \\
& C(\overline{8}, \overline{7}, 2,1)=1, C(\overline{8}, \overline{7}, 6,3)=C(\overline{8}, \overline{9}, 2,3)=3^{-1 / 4} \text {, } \\
& -C(\overline{8}, \overline{9}, 6,3)=C(\overline{8}, \overline{7}, 2,3)=\left(1-\frac{1}{\sqrt{3}}\right)^{1 / 2} \text {, } \\
& C(\overline{9}, \overline{8}, 3,2)=C(\overline{9}, \overline{10}, 3,4)=-C(\overline{9}, \overline{8}, 3,6)=1 \text {, } \\
& C(\overline{10}, \overline{9}, 4,3)=C(\overline{10}, \overline{11}, 4,5)=1 \text {, } \\
& C(\overline{11}, \overline{10}, 5,4)=1 \text {. }
\end{aligned}
$$


It is easy to verify by direct calculation that relation $S$ is verified only at the critical point. (In fact we have chosen the gauge such that $(S)$ is verified.) This shows that $E_{6}$ models can be constructed from $A_{11}$ models via a cell system. We have verified that there exists a matrix $C$ connecting $A_{17}$ to $E_{7}$ and $A_{29}$ to $E_{8}$. There should exist a cell system connecting these graphs. If so, quantum groups, Vertex-IRF correspondence and cell system would permit us to construct all critical CFT with $c \leqq 1$.

(It is indeed true: explicit formula for cell systems connecting $A_{11}$ to $E_{6}, A_{17}$ to $E_{7}$, and $A_{29}$ to $E_{8}$ have been worked out in [DZ].)

\section{Conclusion}

In this article we have developed cell system calculus and shown that it can be applied to lattice models construction. There are lots of questions which are still unanswered:

Cell calculus still remains mysterious. We would like to understand it more abstractly. It would be an important step if one could connect it with paragroups [Oc], which are objects constructed by Ocneanu which generalize the notion of groups, quotient of groups and quantum groups. This could permit us to understand which graphs posess an internal symmetry.

Cell system should also be used to relate partition functions on the torus and local height probabilities of different models. (Steps in this direction have been done in $[\mathrm{FG}]$ in the case of abelian groups.)

It remains to understand why Vertex-IRF correspondence seems to be the lattice analogue of orbifold construction in CFT. It would also be interesting but certainly tedious to construct new models with a different representation of other quantum groups and compare them with known CFT.

\section{Appendix}

1. Proof of Proposition 3. Relation $U$ implies $[(\alpha, \beta)$ being fixed]:

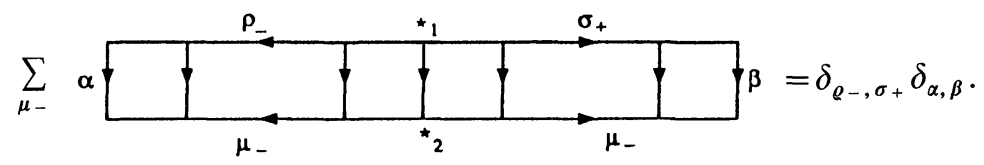

This is done by induction on $l\left(\varrho_{+}\right)$. Indeed,

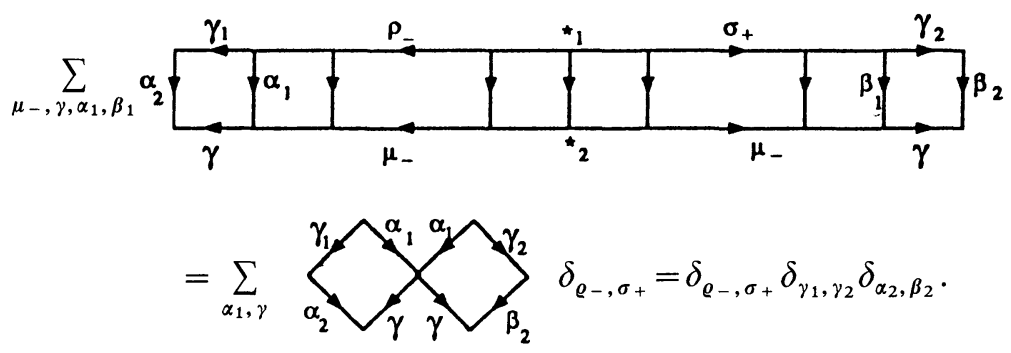


By definition

$$
\varphi^{(N)}\left(\varrho_{+}, \varrho_{-}\right)=
$$

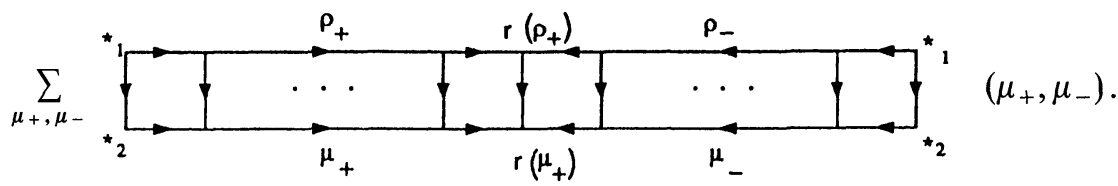

$\varphi^{(N)}\left(\varrho_{+}, \varrho_{-}\right) \varphi^{(N)}\left(\sigma_{+}, \sigma_{-}\right)=$
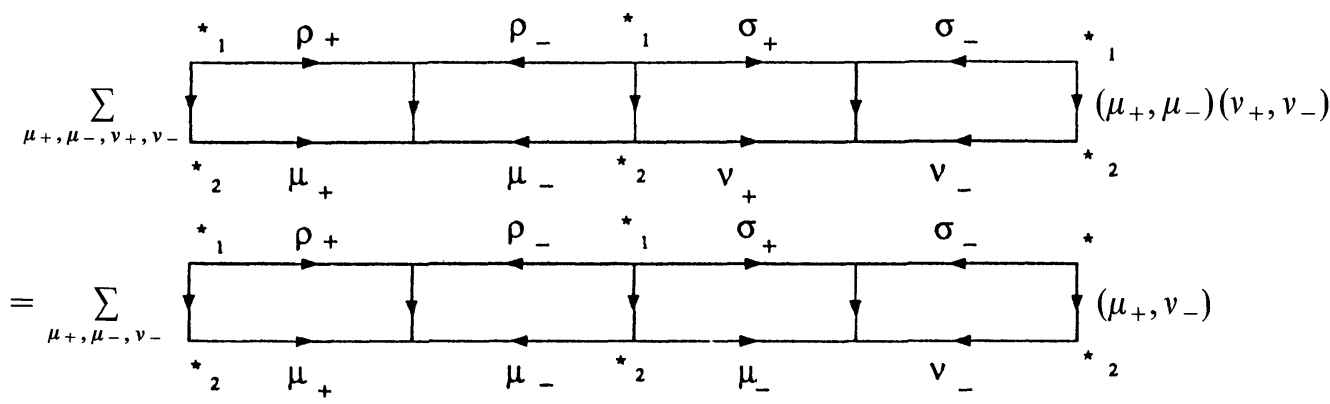

$=\sum_{\mu_{+}, v_{-}}$

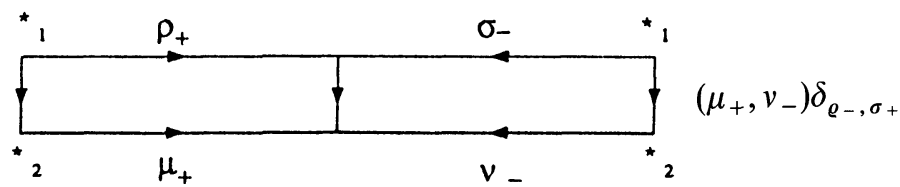

$$
=\varphi^{(N)}\left(\varrho_{+}, \sigma_{-}\right) \delta_{\varrho_{-}, \sigma_{+}}=\varphi^{(N)}\left(\left(\varrho_{+}, \varrho_{-}\right)\left(\sigma_{+}, \sigma_{-}\right)\right) .
$$

This ends the proof of $\varphi^{(N)}$ algebra morphism.

2. Proof of Proposition 4. Relation $T$ implies $\left[\left(\alpha, \alpha^{\prime}\right)\right.$ fixed $]$ :

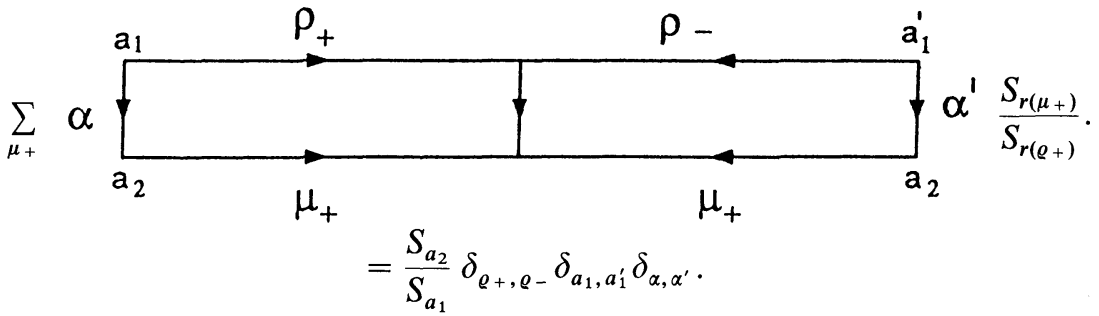

This is done by induction on $l\left(\varrho_{+}\right)$. Indeed,

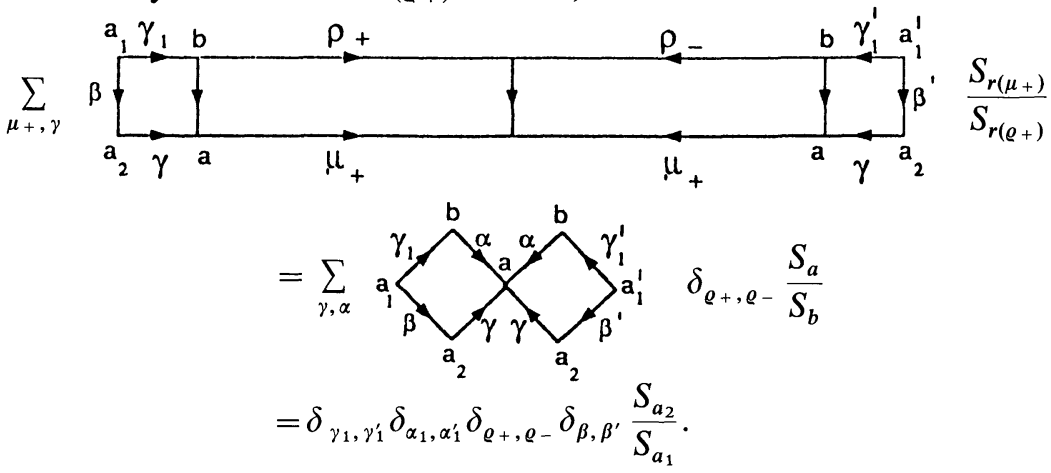


By definition,

$$
\begin{aligned}
& \operatorname{Tr}\left(\varphi^{(N)}\left(\varrho_{+}, \varrho_{-}\right)\right)
\end{aligned}
$$

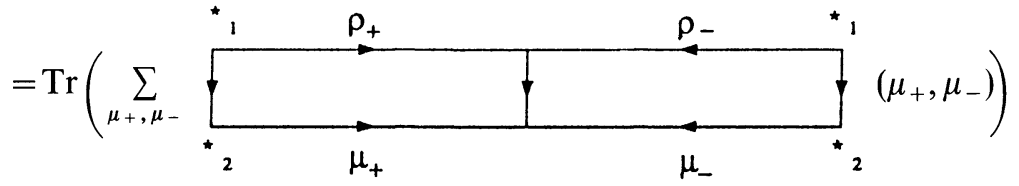

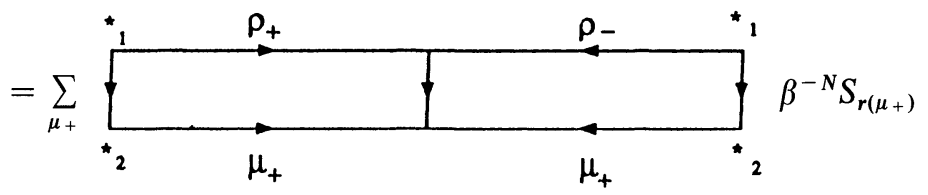

$$
\begin{aligned}
& =\beta^{-N} \delta_{\varrho_{+}, \varrho_{-}} \frac{S_{*_{2}}}{S_{*_{1}}} S_{r\left(\varrho_{+}\right)}=\operatorname{Tr}\left(\varrho_{+}, \varrho_{-}\right) .
\end{aligned}
$$

3. Proof of Proposition 5. We have:

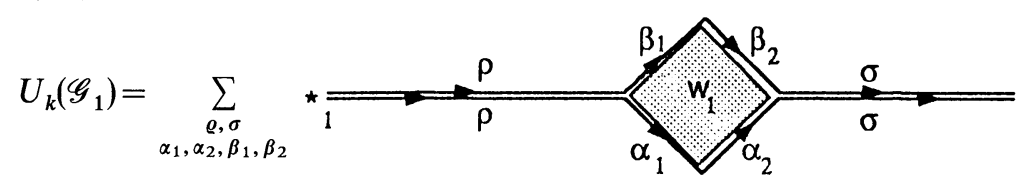

Hence,

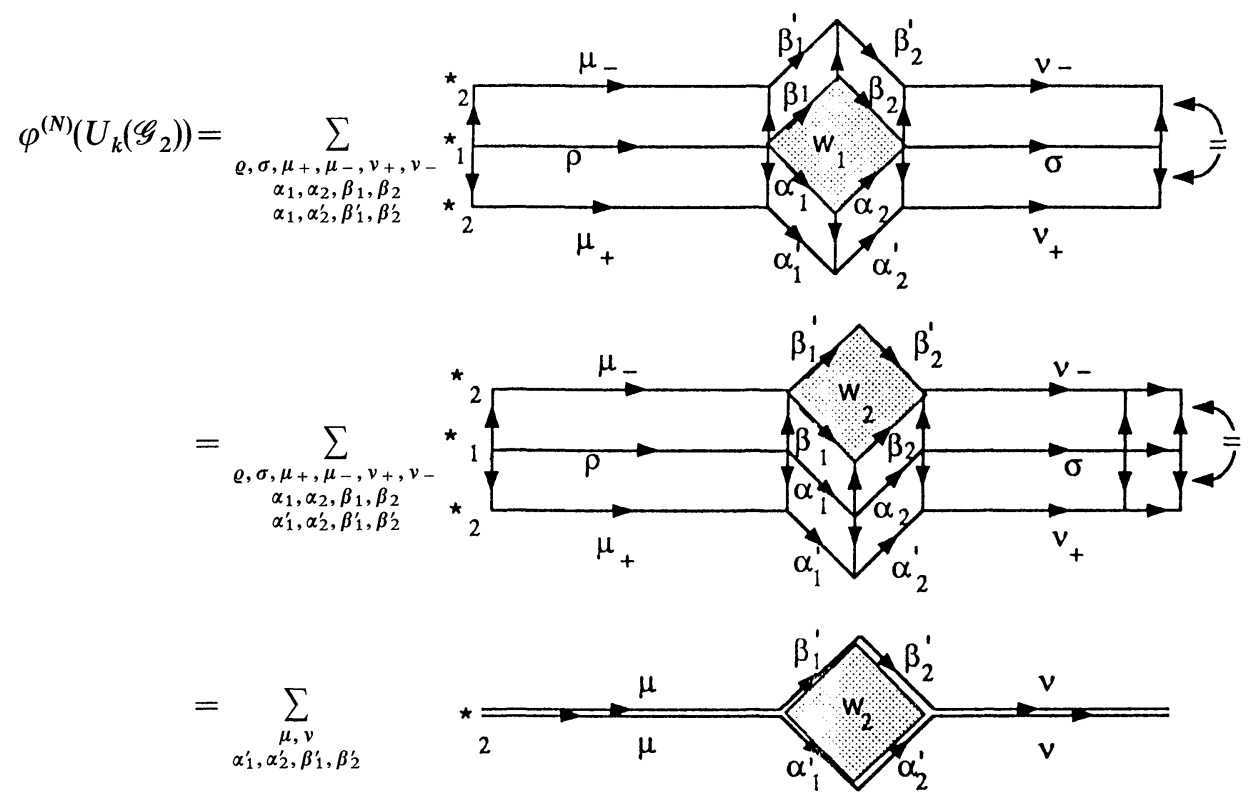

(We have used relation $U$ )

$=U_{k}\left(\mathscr{G}_{2}\right)$. 
4. Proof of Relations $U_{1}$ and $T_{1}$. The left-hand side of relation $U_{1}$ is equal to: $\sum_{\alpha, \beta}\left\langle\varphi_{i j}^{(p)}\left(e_{j}^{(b)}\right) \mid e_{i}^{(a)} \otimes \beta\right\rangle\left\langle e_{i}^{(a)} \otimes \beta \mid \varphi_{i j^{\prime}}^{\left(p^{\prime}\right)}\left(e_{j^{\prime}}^{\left(b^{\prime}\right)}\right)\right\rangle=\left\langle\varphi_{i j}^{(p)}\left(e_{j}^{(b)}\right) \mid \varphi_{i j^{\prime}}^{\left(p^{\prime}\right)}\left(e_{j^{\prime}}^{\left(b^{\prime}\right)}\right)\right\rangle=\delta_{j, j^{\prime}} \delta_{p, p^{\prime}} \delta_{b, b^{\prime}}$, which proves relation $U_{1}$.

Consider the map $\psi: V_{i} \rightarrow V_{i^{\prime}}$ defined by:

$$
\psi(u)=\sum_{\beta, b, a^{\prime}}\left\langle e_{i^{\prime}}^{\left(a^{\prime}\right)} \otimes \beta \mid \varphi_{i^{\prime} j}^{\left(p^{\prime}\right)}\left(e_{j}^{(b)}\right)\right\rangle\left\langle\varphi_{i j}^{(p)}\left(e_{j}^{(b)}\right) \mid u \otimes \beta\right\rangle e_{i^{\prime}}^{\left(a^{\prime}\right)} .
$$

$\psi$ is an intertwiner. Indeed,

$$
\begin{aligned}
\psi\left(\pi_{i}(g) u\right)= & \sum_{\beta, b, a^{\prime}}\left\langle e_{i^{\prime}}^{\left(a^{\prime}\right)} \otimes \beta \mid \varphi_{i^{\prime} j}^{\left(p^{\prime}\right)}\left(e_{j}^{(b)}\right)\right\rangle\left\langle\varphi_{i j}^{(p)}\left(e_{j}^{(b)}\right) \mid \pi_{i}(g) u \otimes \beta\right\rangle e_{i^{\prime}}^{\left(a^{\prime}\right)} \\
= & \sum_{\beta, b, a^{\prime}}\left\langle e_{i^{\prime}}^{\left(a^{\prime}\right)} \otimes \beta \mid \varphi_{i^{\prime} j}^{\left(p^{\prime}\right)}\left(e_{j}^{(b)}\right)\right\rangle\left\langle\left(\pi_{i} \otimes \pi\right)\left(g^{-1}\right) \varphi_{i j}^{(p)}\left(e_{j}^{(b)}\right) \mid u \otimes \pi\left(g^{-1}\right) \beta\right\rangle e_{i^{\prime}}^{\left(a^{\prime}\right)} \\
= & \sum_{\beta, b, a^{\prime}}\left\langle\pi_{i^{\prime}}\left(g^{-1}\right) e_{i^{\prime}}^{\left(a^{\prime}\right)} \otimes \pi\left(g^{-1}\right) \beta \mid \pi_{i^{\prime}} \otimes \pi\left(g^{-1}\right) \varphi_{i^{\prime} j}^{\left(p^{\prime}\right)}\left(e_{j}^{(b)}\right)\right\rangle \\
& \times\left\langle\left(\pi_{i} \otimes \pi\right)\left(g^{-1}\right) \varphi_{i j}^{(p)}\left(e_{j}^{(b)}\right) \mid u \otimes \pi\left(g^{-1}\right) \beta\right\rangle e_{i^{\prime}}^{\left(a^{\prime}\right)} \\
= & \sum_{\beta, b, a^{\prime}}\left\langle\pi_{i^{\prime}}\left(g^{-1}\right) e_{i^{\prime}}^{\left(a^{\prime}\right)} \otimes \pi\left(g^{-1}\right) \beta \mid \varphi_{i^{\prime} j}^{\left(p^{\prime}\right)}\left(\pi_{j}\left(g^{-1}\right) e_{j}^{(b)}\right)\right\rangle \\
& \times\left\langle\varphi_{i j}^{(p)}\left(\pi_{j}\left(g^{-1}\right) e_{j}^{(b)}\right) \mid u \otimes \pi\left(g^{-1}\right) \beta\right\rangle e_{i^{\prime}}^{\left(a^{\prime}\right)} \\
= & \sum_{\beta, b, a^{\prime}}\left\langle e_{i^{\prime}}^{\left(a^{\prime}\right)} \otimes \beta \mid \varphi_{i^{\prime} j}^{\left(p^{\prime}\right)}\left(e_{j}^{(b)}\right)\right\rangle\left\langle\varphi_{i j}^{(p)}\left(e_{j}^{(b)}\right) \mid u \otimes \beta\right\rangle \pi_{i^{\prime}}(g) e_{i^{\prime}}^{\left(a^{\prime}\right)}=\pi_{i^{\prime}}(g) \psi(u) .
\end{aligned}
$$

Consequently, if $i \neq i^{\prime}$ then $\psi=0$ and if $i=i^{\prime}$ then $\psi$ is a $\mathbb{C}$ number. We get

$$
\sum_{\beta, b}\left\langle e_{i^{\prime}}^{\left(a^{\prime}\right)} \otimes \beta \mid \varphi_{i^{\prime} j}^{\left(p^{\prime}\right)}\left(e_{j}^{(b)}\right)\right\rangle\left\langle\varphi_{i j}^{(p)}\left(e_{j}^{(b)}\right) \mid e_{i}^{(a)} \otimes \beta\right\rangle=\lambda\left(p, p^{\prime}, i, j\right) \delta_{i, i^{\prime}} \delta_{a, a^{\prime}} .
$$

When $i^{\prime}=i$ and $a^{\prime}=a$, we have

$$
\sum_{\beta, b, a}\left\langle e_{i}^{(a)} \otimes \beta \mid \varphi_{i j}^{\left(p^{\prime}\right)}\left(e_{j}^{(b)}\right)\right\rangle\left\langle\varphi_{i j}^{(p)}\left(e_{j}^{(b)}\right) \mid e_{i}^{(a)} \otimes \beta\right\rangle=\lambda\left(p, p^{\prime}, i, j\right) \operatorname{dim}\left(\pi_{i}\right) .
$$

The left-hand side of this last equation is equal to

$$
\sum_{b}\left\langle\varphi_{i j}^{(p)}\left(e_{j}^{(b)}\right) \mid \varphi_{i j}^{\left(p^{\prime}\right)}\left(e_{j}^{(b)}\right)\right\rangle=\delta_{p, p^{\prime}} \operatorname{dim}\left(\pi_{j}\right) .
$$

This proves relation $T_{1}$.

Proof of Vertex-IRF Correspondence. The right-hand side of ( $V$-IRF) is equal to:

$$
\begin{aligned}
& \sum_{\beta_{1}, \beta_{2}, b}\left\langle\varphi_{i j}^{\left(p_{1}\right)}\left(e_{j}^{(b)}\right) \mid e_{i}^{(a)} \otimes \beta_{1}\right\rangle\left\langle\varphi_{j k}^{\left(p_{2}\right)}\left(e_{k}^{(c)}\right) \mid e_{j}^{(b)} \otimes \beta_{2}\right\rangle\left\langle\beta_{1} \otimes \beta_{2}|R| \alpha_{1} \otimes \alpha_{2}\right\rangle \\
& =\sum_{\beta_{1}, \beta_{2}, b}\left\langle\left(\varphi_{i j}^{\left(p_{1}\right)} \otimes 1\right) \varphi_{i j}^{\left(p_{2}\right)}\left(e_{k}^{(c)}\right) \mid \varphi_{i j}^{\left(p_{1}\right)}\left(e_{j}^{(b)}\right) \otimes \beta_{2}\right\rangle \\
& \quad \times\left\langle\varphi_{i j}^{\left(p_{1}\right)}\left(e_{j}^{(b)}\right) \mid e_{i}^{(a)} \otimes \beta_{1}\right\rangle\left\langle\beta_{1} \otimes \beta_{2}|R| \alpha_{1} \otimes \alpha_{2}\right\rangle .
\end{aligned}
$$

Introducing the projector

$$
P_{i j}^{\left(p_{1}\right)}=\sum_{b}\left|\varphi_{i j}^{\left(p_{1}\right)}\left(e_{j}^{(b)}\right)\right\rangle\left\langle\varphi_{i j}^{\left(p_{1}\right)}\left(e_{j}^{(b)}\right)\right|,
$$

the last expression is equal to:

$$
\begin{aligned}
& =\sum_{\beta_{1}, \beta_{2}}\left\langle\left(\varphi_{i j}^{\left(p_{1}\right)} \otimes 1\right) \varphi_{j k}^{\left(p_{2}\right)}\left(e_{k}^{(c)}\right)\left|\left(P_{i j}^{\left(p_{1}\right)} \otimes 1\right)\right| e_{i}^{(a)} \otimes \beta_{1} \otimes \beta_{2}\right\rangle\left\langle\beta_{1} \otimes \beta_{2}|R| \alpha_{1} \otimes \alpha_{2}\right\rangle \\
& =\left\langle\left(\varphi_{i j}^{\left(p_{1}\right)} \otimes 1\right) \varphi_{j k}^{\left(p_{2}\right)}\left(e_{k}^{(c)}\right)|1 \otimes R| e_{i}^{(a)} \otimes \alpha_{1} \otimes \alpha_{2}\right\rangle .
\end{aligned}
$$


The left-hand side of equation ( $V$-IRF) is equal to:

$$
\begin{gathered}
\sum_{j^{\prime}, p_{1}^{\prime}, p_{2}^{\prime}, b^{\prime}}\left\langle\left(\varphi_{i j}^{\left(p_{1}\right)} \otimes 1\right) \varphi_{j k}^{\left(p_{2}\right)}\left(e_{k}^{(c)}\right)|1 \otimes R| u\right\rangle, \\
u=\sum_{j^{\prime}, p_{1}^{\prime}, p_{2}^{\prime}, b^{\prime}}\left(\varphi_{i j^{\prime}}^{\left(p_{1}^{\prime}\right)} \otimes 1\right) \varphi_{j^{\prime} k}^{\left(p_{2}^{\prime}\right)}\left(e_{k}^{(c)}\right)\left\langle\varphi_{i j^{\prime}}^{\left(p_{1}^{\prime}\right)}\left(e_{j^{\prime}}^{\left(b^{\prime}\right)}\right) \mid e_{i}^{(a)} \otimes \alpha_{1}\right\rangle\left\langle\varphi_{j^{\prime} k}^{\left(p^{\prime}\right)}\left(e_{k}^{(c)}\right) \mid e_{j^{\prime}}^{\left(b^{\prime}\right)} \otimes \alpha_{2}\right\rangle, \\
u=\sum_{j^{\prime}, p_{1}^{\prime}, p_{2}^{\prime}, b^{\prime}}\left(\varphi_{i j^{\prime}}^{\left(p^{\prime}\right)} \otimes 1\right) \varphi_{j^{\prime} k}^{\left(p^{\prime}\right)}\left(e_{k}^{(c)}\right)\left\langle\left(\varphi_{i j^{\prime}}^{\left(p^{\prime}\right)} \otimes 1\right) \varphi_{j^{\prime} k}^{\left(p_{2}^{\prime}\right)}\left(e_{k}^{(c)}\right) \mid \varphi_{i j^{\prime}}^{\left(p^{\prime}\right)}\left(e_{j^{\prime}}^{\left(b^{\prime}\right)}\right) \otimes \alpha_{2}\right\rangle \\
\quad \times\left\langle\varphi_{i j^{\prime}}^{\left(p^{\prime}\right)}\left(e_{j^{\prime}}^{\left(b^{\prime}\right)}\right) \mid e_{i}^{(a)} \otimes \alpha_{1}\right\rangle \\
=\sum_{j^{\prime}, p_{1}^{\prime}, p_{2}^{\prime}}\left(\varphi_{i j^{\prime}}^{\left(p_{1}^{\prime}\right)} \otimes 1\right) \varphi_{j^{\prime} k}^{\left(p_{2}^{\prime}\right)}\left(e_{k}^{(c)}\right)\left\langle\left(\varphi_{i j^{\prime}}^{\left(p^{\prime}\right)} \otimes 1\right) \varphi_{j^{\prime} k}^{\left(p_{2}^{\prime}\right)}\left(e_{k}^{(c)}\right)\left|P_{i j^{\prime}}^{\left(p^{\prime}\right)} \otimes 1\right| e_{i}^{(a)} \otimes \alpha_{1} \otimes \alpha_{2}\right\rangle \\
=P_{c}\left(e_{i}^{(a)} \otimes \alpha_{1} \otimes \alpha_{2}\right),
\end{gathered}
$$

where $P_{c}$ is the projector defined by

$$
P_{c}=\sum_{j^{\prime}, p_{1}^{\prime}, p_{2}^{\prime}}\left|\left(\varphi_{i j^{\prime}}^{\left(p^{\prime}\right)} \otimes 1\right) \varphi_{j^{\prime} k}^{\left(p_{2}^{\prime}\right)}\left(e_{k}^{(c)}\right)\right\rangle\left\langle\left(\varphi_{i j^{\prime}}^{p_{1}^{\prime}} \otimes 1\right) \varphi_{j^{\prime} k}^{\left(p^{\prime}\right)}\left(e_{k}^{(c)}\right)\right| .
$$

The reader will verify that $[R, \pi \otimes \pi(g)]=0 \forall g \in G$ implies $\left[P_{c}, 1 \otimes R\right]=0$. Consequently the left-hand side of equation $(V$-IRF) is equal to:

$$
\begin{aligned}
& \left\langle\left(\varphi_{i j}^{\left(p_{1}\right)} \otimes 1\right) \varphi_{j k}^{\left(p_{2}\right)}\left(e_{k}^{(c)}\left|(1 \otimes R) P_{c}\right| e_{i}^{(a)} \otimes \alpha_{1} \otimes \alpha_{2}\right\rangle\right. \\
& \quad=\left\langle\left(\varphi_{i j}^{\left(p_{1}\right)} \otimes 1\right) \varphi_{i j}^{\left(p_{2}\right)}\left(e_{k}^{(c)}\right)\left|P_{c}(1 \otimes R)\right| e_{i}^{(a)} \otimes \alpha_{1} \otimes \alpha_{2}\right\rangle \\
& =\left\langle\left(\varphi_{i j}^{\left(p_{1}\right)} \otimes 1\right) \varphi_{j k}^{\left(p_{2}\right)}\left(e_{k}^{(c)}\right)|(1 \otimes R)| e_{i}^{(a)} \otimes \alpha_{1} \otimes \alpha_{2}\right\rangle .
\end{aligned}
$$

This ends the proof of the Vertex-IRF correspondence.

Acknowledgements. The author warmfully thanks V. Pasquier for private communications and preprints, as well as D. Arnaudon, M. Bauer, J. Lascoux, and M. Petropoulos for encouragement and discussions.

\section{References}

[ABF] Andrews, G.E., Baxter, R.J., Forester, P.J.: J. Stat. Phys. 35, 193 (1983)

[AuY] Au-Yang, H., MacCoy, B., Perk, J.H.H., Tang, S., Yan, M.: Commuting transfer matrices of star triangle equations with genus greater than 1. Phys. Lett. A 123, 219 (1987)

[AW] Akutsu, Y., Wadati, M.: Commun. Math. Phys. 117, 243 (1988)

[Ba.1] Baxter, R.J.: Exactly solved models in statistical mechanics. New York: Academic Press 1982

[Ba.2] Baxter, R.J.: Ann. Phys. 76, 25 (1973)

[Ba.3] Baxter, R.J., Perk, J.H.H., Au-Yang, H.: New solutions of the star triangle relations for the chiral potts model. Phys. Lett. A 128, 138 (1988)

[BPZ] Belavin, A.A., Polyakov, A.M., Zamolodchikov, A.B.: Nucl. Phys. B 241, 333 (1984)

[BW] Birman, J., Wenzl, H.: Braids, Link polynomials and a new algebra. Columbia University preprint 1987

[CIZ] Cappelli, A., Itzykson, C., Zuber, J.B.: Commun. Math. Phys. 113, 1 (1987)

[DJKMO] Date, E., Jimbo, M., Kuniba, A., Miwa, T., Okado, M.: Nucl. Phys. B 290 [FS 20], 231 (1987)

[Dr] Drinfeld, V.G.: Quantum groups, Proc. I.C.M. Berkeley 1986

[DGH] Dixon, L., Ginsparg, P., Harvey, J.: Nucl. Phys. B 306, 470 (1988)

[DSZ] DiFrancesco, P., Saleur, H., Zuber, J.B.: Nucl. Phys. B 300 [FS 22], 393 (1988) 
[DZ] DiFrancesco, Ph., Zuber, J.B.: $S U(N)$ Lattice integrable models associated with graphs. S.Ph-T/89/92 Juin 1989 preprint

[FG] Fendley, P., Ginsparg, P.: Non-critical orbifolds, Harvard preprint 1989

[Ga] Gantmacher, F.R.: The theory of Matrices 2. Chelsea Publishing Company 1959

[Gi.1] Ginsparg, P.: Applied conformal field theory. Les Houches Proceedings 1988

[Gi.2] Ginsparg, P.: Nucl. Phys. B 295, 153 [FS 21] (1988)

[Ji] Jimbo, M.: Commun. Math. Phys. 102, 537 (1986)

Jimbo, M.: Lett. Math. Phys. 11, 247 (1986)

[Jo.1] Jones, V.F.R.: Index for subfactors 1983. Invent. Math. 72, 1 (1983)

[Jo.2] Jones, V.F.R.: Hecke algebra, representations of Braid group and Link Polynomials. Ann. Math. 126, 335 (1987)

[KRS] Kulish, P.P., Reshetikhin, N.Y., Sklyanin, E.K.: Lett. Math. Phys. 5, 393 (1981)

[McK] MacKay, J.: Cartan matrices, finite groups of quaternions and Kleinian singularities. Proc. Am. Math. Soc. 1981

[MS] Moore, G., Seiberg, N.: Classical and quantum CFT. Commun. Math. Phys. 123, 177 (1989)

[Oc] Ocneanu, A.: Quantized groups, string algebras and Galois theory for algebras in Operator algebra and applications. Lond. Math. Soc. Lecture note Series 136

[Pa.1] Pasquier, V.: Nucl. Phys. B 285 [FS 19], 162 (1987)

[Pa.2] Pasquier, V.: Commun. Math. Phys. 118, 355 (1988)

[Pa.3] Pasquier, V.: Thesis unpublished 1988

[Pa.4] Pasquier, V.: J. Phys. A Math. Gen. 20, L21 (1987)

[Pa.5] Pasquier, V.: J. Phys. A Math. Gen. 20, L1229 (1987)

[Sk] Sklyanin, V.G.: Funct. Anal. Appl. 17, 223 (1983)

[Su] Sunder, V.S.: An invitation to von Neumann algebras. Berlin, Heidelberg, New York: Springer 1987

Communicated by K. Gawedzki

Received June 29, 1989; in revised form July 19, 1989 